\title{
Preview of Comet C/2021 A1 (Leonard) and Its Encounter with Venus
}

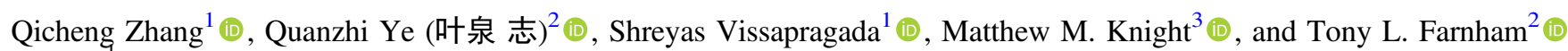 \\ ${ }^{1}$ Division of Geological and Planetary Sciences, California Institute of Technology, Pasadena, CA 91125, USA; qicheng@ cometary.org \\ ${ }_{3}^{2}$ Department of Astronomy, University of Maryland, College Park, MD 20742, USA \\ ${ }^{3}$ Department of Physics, United States Naval Academy, Annapolis, MD 21402, USA \\ Received 2021 June 20; revised 2021 July 20; accepted 2021 July 24; published 2021 October 13
}

\begin{abstract}
Long-period comet C/2021 A1 (Leonard) will approach Venus to within 0.029 au on 2021 December 18 and may subsequently graze the planet with its dust trail less than 2 days later. We observed $\mathrm{C} / 2021 \mathrm{~A} 1$ with the Lowell Discovery Telescope on 2021 January 13 and March 3, as well as with the Palomar Hale Telescope on 2021 March 20, while the comet was inbound at heliocentric distances of $r=4.97,4.46$, and 4.28 au, respectively. Tail morphology suggests that the dust is optically dominated by $\sim 0.1-1 \mathrm{~mm}$ radius grains produced in the prior year. Neither narrowband imaging photometry nor spectrophotometry reveal any definitive gas emission, placing $3 \sigma$ upper bounds on $\mathrm{CN}$ production of $\lesssim 10^{23} \mathrm{molec}^{-1}$ at both of the latter two epochs. Trajectory analysis indicates that large $(\gtrsim 1 \mathrm{~mm})$ grains ejected at extremely large heliocentric distances $(r \gtrsim 30 \mathrm{au})$ are most strongly favored to reach Venus. The flux of such meteors on Venus, and thus their potential direct or indirect observability, is highly uncertain, as the comet's dust production history is poorly constrained at these distances but will likely fall well below the meteor flux from comet C/2013 A1 (Siding Spring)'s closer encounter to Mars in 2014 and thus poses a negligible risk to any spacecraft in orbit around Venus. Dust produced in previous apparitions will not likely contribute substantially to the meteor flux, nor will dust from any future activity apart from an unlikely high-speed $\left(\gtrsim 0.5 \mathrm{~km} \mathrm{~s}^{-1}\right.$ ) dust outburst prior to the comet reaching $r \approx 2$ au in 2021 September.
\end{abstract}

Unified Astronomy Thesaurus concepts: Comet tails (274); Long period comets (933); Meteor trails (1036); Venus (1763)

\section{Introduction}

Long-period comet C/2021 A1 (Leonard) was discovered by G. J. Leonard on 2021 January 3 as part of the Catalina Sky Survey (Leonard et al. 2021a). Prediscovery observations were subsequently located to extend the astrometric observation arc back to 2020 April 11 and indicated that it would approach to within 0.029 au of Venus on 2021 December 18 (Leonard et al. 2021b), a distance closer than that of any reliably constrained encounter of a long-period comet to Earth. ${ }^{4}$ More remarkably, Venus will pass only $\sim 50,000 \mathrm{~km}$ - the minimum orbit intersection distance (MOID)-from the comet's heliocentric orbit on 2021 December 19 at a point only 3 days behind the nucleus. This passage is notable because the principal action of solar radiation pressure on dust grains ejected from a comet nucleus is to confine them to a size-sorted dust fan, with the smallest grains (radius $a_{d} \sim 1 \mu \mathrm{m}$ ) rapidly dispersed in the antisunward direction and the largest grains $\left(a_{d} \gtrsim 1 \mathrm{~mm}\right)$ more slowly concentrated into a narrow trail along the comet's heliocentric orbit, lagging behind the nucleus (Finson \& Probstein 1968). The exceptionally close approach of Venus to a portion of the comet's orbit close behind the nucleus therefore raises the possibility that large dust grains from $\mathrm{C} /$ 2021 A1 may reach Venus to produce a meteor shower.

Meteors are more commonly associated with short-period comets, which rapidly fill their physically smaller orbits over many revolutions with dust trails that may be directly observed (e.g., Sykes \& Walker 1992) or otherwise inferred from meteor outbursts when Earth crosses their orbits (e.g., Kresak 1993). Past trails of longer-period comets are more sparsely distributed and thinly stretched over their larger orbits, although meteors from such trails are also occasionally detected (e.g., Lyytinen \&

\footnotetext{
4 https://www.minorplanetcenter.net/iau/lists/ClosestComets.html
}

Jenniskens 2003). Beech (1998), Christou (2010), and others have additionally investigated such periodic meteoroid streams for their potential to generate annual meteor showers on Venus, although such meteor showers have yet to be clearly observed.

Although not a dynamically new comet, C/2021 A1 follows a very large orbit with inbound barycentric semimajor axis $a_{\text {in }} \approx 1900 \mathrm{au}$, corresponding to a dynamical lifetime on the order of one orbital period (Królikowska \& Dybczyński 2017), which precludes the comet from having developed a discernible, periodic meteoroid stream (Jenniskens et al. 2021). Rather, we are interested in the potential for meteors produced in the ongoing apparition-while the dust is still concentrated behind the nucleus-which is a far more rare occurrence that requires not only a close approach of a comet but one immediately followed by the planet crossing the comet's orbit behind the nucleus. A nearly as close 0.031 au encounter of C/ 1983 H1 (IRAS-Araki-Alcock) with Earth-the closest known of a long-period comet to Earth - thus produced minimal enhancement in meteor activity, as Earth crossed ahead of rather than behind the nucleus, missing the dust trail (Ohtsuka 1991). Likewise, the 0.07 au encounter of C/ 1976 E1 (Bradfield) with Venus-the previous closest by a long-period comet to Venus reported by JPL SBDB ${ }^{5}$ - likely produced no meteor activity, as Venus passed far interior to the comet's orbit, where no recent dust from the comet could plausibly reach.

Nonetheless, the favorability of C/2021 A1's encounter with Venus is not unprecedented. Most notably, comet C/2013 A1 (Siding Spring) passed $140,000 \mathrm{~km}$ from Mars on 2014 October 19 (Farnocchia et al. 2016). Following this encounter, Mars crossed the dust trail (MOID 30,000 km)

\footnotetext{
5 https://ssd-api.jpl.nasa.gov/doc/cad.html
} 
at a point less than $3 \mathrm{hr}$ behind the nucleus, intercepting a sizable fraction of the large, $1-10 \mathrm{~mm}$ grains released from the nucleus earlier at a heliocentric distance of $r \sim 22.5 \mathrm{au}$ (Farnocchia et al. 2014). Ultraviolet spectroscopy by the MAVEN spacecraft in orbit around Mars at the time subsequently revealed the appearance of a temporary metallic vapor layer in the atmosphere with a density consistent with $\sim 10^{4} \mathrm{~kg}$ of deposited dust (Schneider et al. 2015), while associated perturbations to the ionosphere were independently detected by multiple other spacecraft and instruments (Benna et al. 2015; Restano et al. 2015; Sánchez-Cano et al. 2020).

In the following sections, we present early imagery and spectroscopy of C/2021 A1 to provide an initial characterization of the comet and enable a preliminary comparison with other comets. We then discuss the orbital setup and both the dynamical and physical requirements for dust to reach Venus and compare these results with those of the prior encounter of C/2013 A1 with Mars. We also briefly speculate on the prospects for detecting meteors on Venus either by direct observation or indirectly through the presence of a meteoritic layer or associated ionospheric perturbations as seen on Mars.

\section{Observations}

We observed C/2021 A1 with the Lowell Discovery Telescope (LDT) on 2021 January 13 and 2021 March 3 for optical broadband and narrowband imaging and with the Palomar Hale Telescope (P200) on 2021 March 20 for nearinfrared $(J)$ imaging and optical spectroscopy. These observations and the photometry derived from them are summarized in Table 1, with associated images of the comet at all epochs shown in Figure 1.

\subsection{Lowell Discovery Telescope}

We observed the comet using the Large Monolithic Imager (LMI; Massey et al. 2013) on LDT. The LMI has a field of view of 12 ! $3 \times 12$. 3 and a pixel scale of 0 ". 36 after an on-chip $3 \times 3$ binning. For the 2021 January 13 run, we collected three usable images through the $V R(V+R$; introduced by Jewitt et al. 1996) filter only, each with a $300 \mathrm{~s}$ exposure. For the 2021 March 3 run, we collected two sets of images: broadband imagery in $r^{\prime}$, as well as narrowband imagery through the $\mathrm{CN}$, blue continuum (BC), and red continuum (RC) filters of the HB set (Farnham et al. 2000). The center wavelengths of these filters are 387,445 , and $713 \mathrm{~nm}$, respectively, selected to contain the violet $\mathrm{CN}(\Delta v=0)$ emission bands and nearly gasfree continuum points. We began and ended the observation with one $30 \mathrm{~s} r^{\prime}$ image and cycled through $\mathrm{RC}, \mathrm{CN}$, and $\mathrm{BC}$ three times, with a $180 \mathrm{~s}$ exposure for each filter each time. This strategy counters brightness variations in time, including from changing airmass and any passing cirrus, although we note that the sky was clear during the observation. We also dithered the pointing between each exposure in order to mitigate detector defects such as dead pixels and pixel-to-pixel variation.

We then performed bias subtraction and flat-field correction on the images collected from both nights. Pixels below the 5th and above the 95th percentiles, excluding a region of $5^{\prime \prime}$ in radius centered on the comet $\left(10^{\prime \prime}\right.$ for the $V R$ images from 2021 January 13 due to the images being deeper), were masked to remove background stars. Frames were then mediancombined into the final stacked images for all filters.
The $V R$ and $r^{\prime}$ images were photometrically calibrated using the Pan-STARRS1 DR1 catalog (Chambers et al. 2016), with $V R$ approximated as $r^{\prime}$. The narrowband images were photometrically calibrated using the HB magnitudes of eight field stars computed from their spectra observed by LAMOST (Cui et al. 2012). These HB magnitudes were derived by convolving the transmission profile of each $\mathrm{HB}$ filter with the LAMOST spectrum of the star calibrated using the PanSTARRS1 DR1 catalog, since the LAMOST catalog does not provide absolutely calibrated fluxes. To exclude variable stars and other poor-quality spectra, we only used stars with a broadband color error-estimated as the standard deviation of the zero-point magnitudes of the spectrum measured for $g^{\prime}, r^{\prime}$, and $i^{\prime}$-below $0.1 \mathrm{mag}$. We also collected images of $\mathrm{HB}$ standards during our observation but encountered unexpected instrumental behavior that prevented us from using these images to perform absolute calibration.

\subsection{Palomar Hale Telescope}

We used the Wide-field Infrared Camera (WIRC; Wilson et al. 2003) on P200 to collect $J$-band imagery of the comet. We took $46 \times 60 \mathrm{~s}$ exposures spread over a nine-point $(3 \times 3)$ square dither pattern with a grid spacing of $90^{\prime \prime}$. We performed background subtraction for frames at each dither point by using the median of all frames at the other eight dither positions as the background frame. We then applied flat-field correction with dome flats taken at the beginning of the night, then astrometrically and photometrically solved each frame with field stars from the Gaia EDR3 (Gaia Collaboration et al. 2021) and Two Micron All Sky Survey (2MASS; Skrutskie et al. 2006) catalogs, respectively, before aligning and stacking all frames on the position of the comet.

We also used the double spectrograph (DBSP; Oke \& Gunn 1982) to collect low-resolution spectroscopy of the comet. We used a standard configuration capturing wavelengths of $\sim 300-550 \mathrm{~nm}$ on the blue side and $\sim 550-1100 \mathrm{~nm}$ on the red side, as well as a wide $10^{\prime \prime}$ slit, in order to maximize the signal from the comet and improve sensitivity to any diffuse gas emission, and we took $4 \times 300 \mathrm{~s}$ exposures through both channels. The slit was oriented in the east-west direction, roughly perpendicular to the tail. As the slit is much wider than the typical atmospheric dispersion over the covered wavelength range, dispersion slit losses are negligible, irrespective of slit orientation. We additionally observed the nearby $\left(12^{\circ}\right.$ away) spectrophotometric standard star Grw +705824 (airmass 1.35) and took $4 \times 60 \mathrm{~s}$ exposures with the same configuration immediately prior to observing the comet (airmass 1.14) for flux and sky calibration.

We used PypeIt (Prochaska et al. 2020) to extract a $10^{\prime \prime}$ wide region centered on the comet for spectrophotometry, corresponding to a $10^{\prime \prime} \times 10^{\prime \prime}$ box aperture. Figure 2 shows the summed and fully calibrated spectrum, binned to $3 \mathrm{~nm}$ resolution, as well as an overlay of the slit and extraction aperture over the WIRC image. The solar spectrum of Meftah et al. (2018) is provided as a comparison and was divided into the absolute comet spectrum to produce a relative reflectance spectrum. Flux calibration produced a number of artifacts near major telluric absorption features on the red side, likely due to the difference in airmass and brightness profile between the calibration star and comet, and the most prominent of these artifacts have been masked out to aid in determination of the dust color. A detector artifact on the red camera near $550 \mathrm{~nm}$ 
Table 1

Observations of C/2021 A1

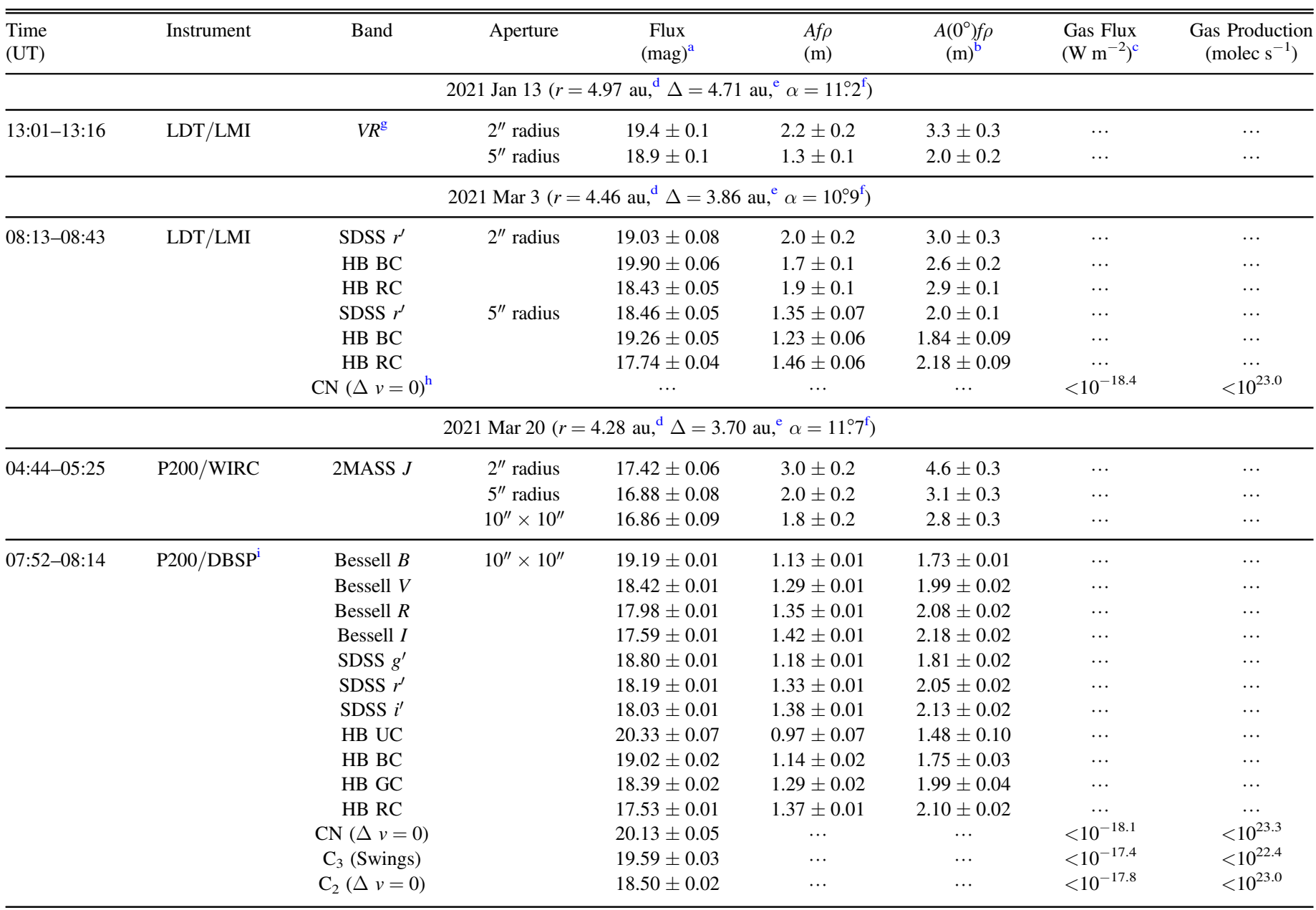

Notes.

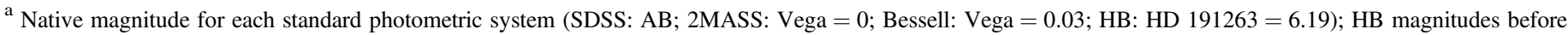
continuum subtraction for gas emission bands.

${ }^{\mathrm{b}}$ Converted from observed Af $\rho$ with the Schleicher-Marcus phase function (Schleicher \& Bair 2011).

${ }^{\mathrm{c}}$ Full-band continuum-subtracted gas flux. Upper limits are $3 \sigma$.

d Heliocentric distance.

e Geocentric distance.

${ }^{\mathrm{f}}$ Phase angle.

g Calibrated as SDSS $r^{\prime}$.

${ }^{\mathrm{h}}$ Derived from aperture differencing (see Section 2.4) and converted to $5^{\prime \prime}$ radius equivalent aperture.

${ }^{\mathrm{i}}$ Relative uncertainties given for DBSP colors. Absolute flux and Af $\rho$ for stated aperture estimated to be $\pm 5 \%$.

was also masked out. We used PypeIt's standard backgroundsubtraction routine, which measures the sky outside the $10^{\prime \prime}$ extraction box of our C/2021 A1 spectrum, except for the wavelength range $380-520 \mathrm{~nm}$, where we instead subtracted a scaled version of the measured Grw +705824 sky background in order to avoid subtraction of any spatially extended gas emission in this wavelength range. We do not consider any gas emission features beyond this range, and the contemporaneous imagery shows the dust coma to be clearly contained within the $10^{\prime \prime}$ box, so no features of interest should be included in the subtracted background. Dust colors and gas production limits are discussed further in Sections 2.3 and 2.4, respectively.

\subsection{Dust Properties}

Under the formalism of Finson \& Probstein (1968), the spatial position of a dust grain released from the nucleus at zero velocity $\left(v_{d}=0 \mathrm{~m} \mathrm{~s}^{-1}\right)$ is uniquely determined by a grid of two properties: (1) the time since the grain was released, $\tau$, and (2) the size parameter $\beta$, defined as the ratio of force of solar radiation pressure acting on the grain to that of solar gravitation. For typical low-albedo grains of a bulk density $\rho_{d} \sim 0.5 \mathrm{~g} \mathrm{~cm}^{-3}$, the effective grain radius $a_{d}$ is approximately related to $\beta$ by the inverse relation $a_{d} \sim 1 \mu \mathrm{m} / \beta$. We note that estimates of the mean $\rho_{d}$ vary substantially, with values reported in the range of $\sim 0.1-4 \mathrm{~g} \mathrm{~cm}^{-3}$ (Lasue et al. 2009), with individual comets often producing a mixture of both compact and fluffy grains (Kolokolova et al. 2018). Differences 


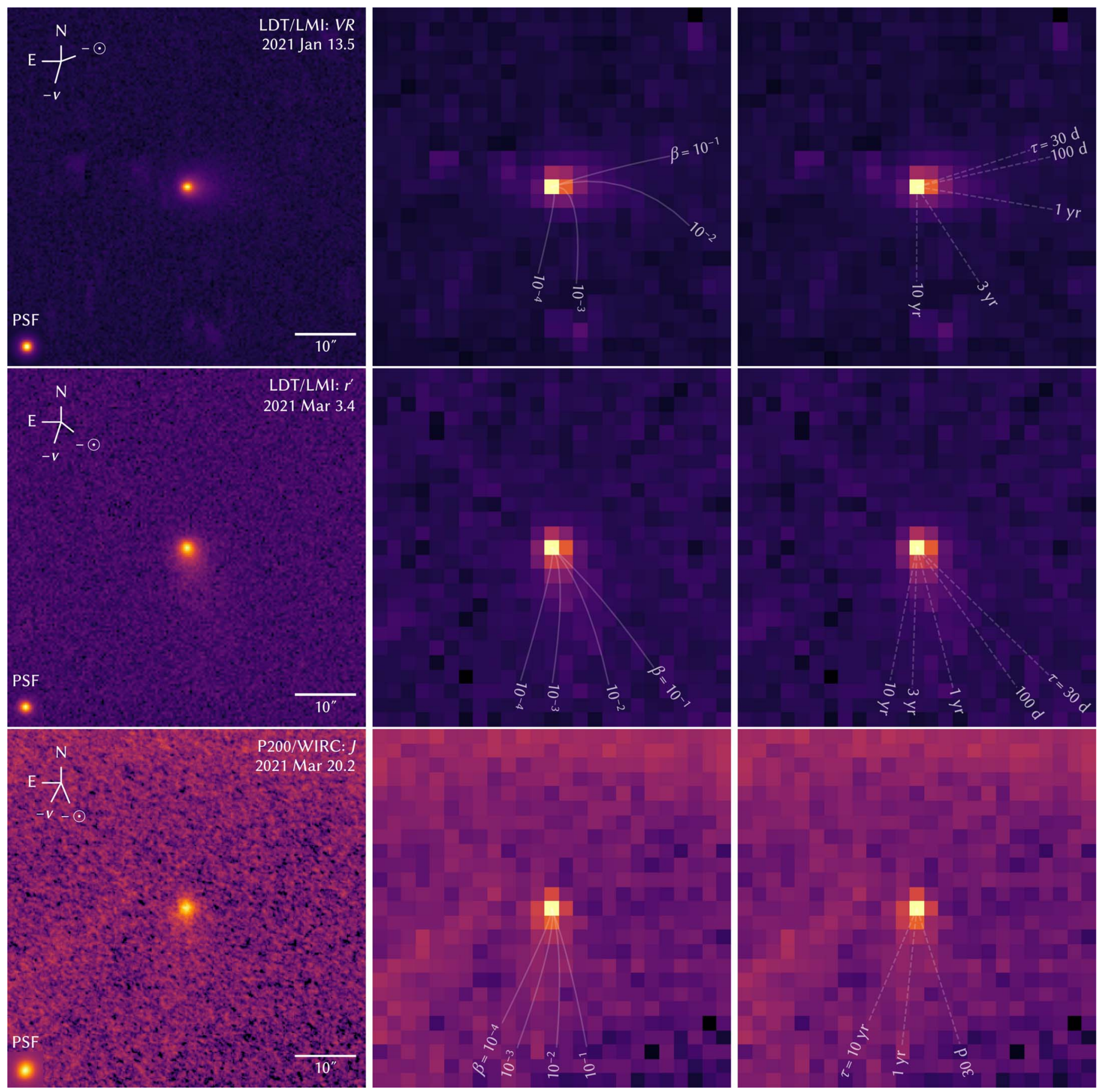

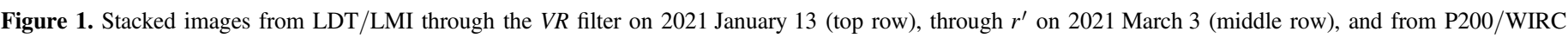

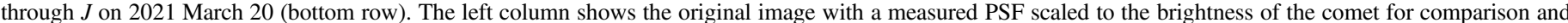

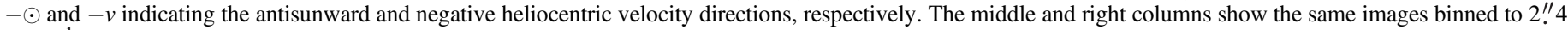

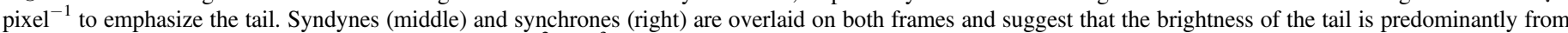

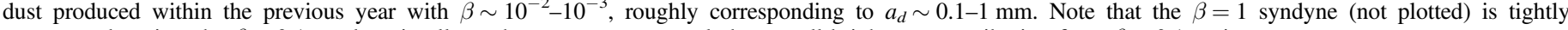
compressed against the $\beta=0.1$ syndyne in all epochs, so we cannot exclude a small brightness contribution from $\beta>0.1$ grains

in $\rho_{d}$ do not affect our results, except in this rough conversion between $\beta$ and $a_{d}$.

All stacked images in Figure 1 are accompanied by binned and stretched versions that emphasize the low surface brightness tail and are overlaid with a selection of syndynes and synchrones-curves where $v_{d}=0$ dust grains of equal $\beta$ and equal $\tau$ fall, respectively. The actual nonzero $v_{d}$ means that the dust with these properties will only approximately fall along these curves and may be biased to one side by anisotropic dust production, but comparison of tail morphology with respect to the grid of $\beta$ and $\tau$ still enables a useful, if rudimentary, initial characterization of the dust properties in the tail well away from the coma.

The tail within the observed $\sim 30^{\prime \prime}$ of the coma is cleanly confined to $\beta>10^{-4}$, with the surface brightness maximum near $\beta \sim 10^{-2}-10^{-3}$ at $\tau \lesssim 1 \mathrm{yr}$, corresponding to $a_{d} \sim 0.1-1 \mathrm{~mm}$. This dust is much larger than typically observed from comets nearer to the Sun $(r \sim 1 \mathrm{au})$, which are 

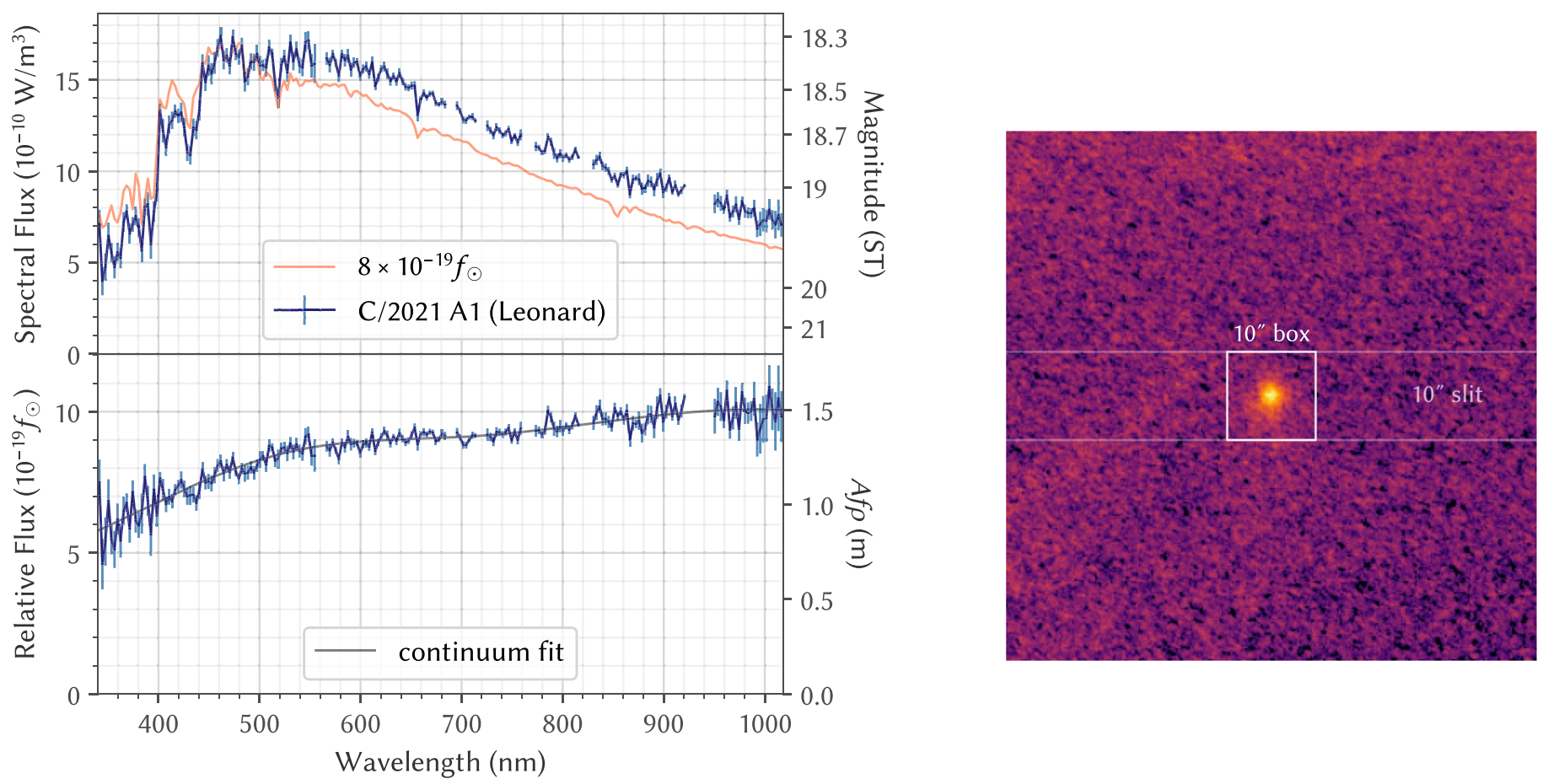

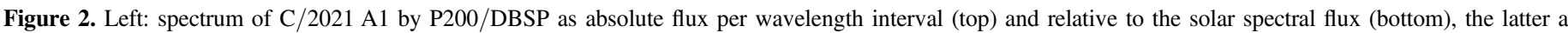

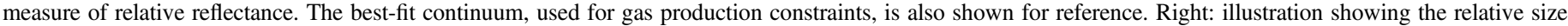
and orientation of the $10^{\prime \prime}$ slit and extraction box aperture overlaid on the P200/WIRC image from Figure 1.

usually brightest in the $\beta \sim 0.1-1$ range (Fulle 2004). However, it is comparable with the optically dominant $\beta \sim 10^{-2}$ dust grains observed from other comets at similar $r$, including $\mathrm{C} /$ 2013 A1 (Ye \& Hui 2014).

We note that the dust that is optically most prominent in the tail is not necessarily representative of the mass distribution of the dust at any point in time. In particular, while much of the observed $\beta \sim 10^{-2}-10^{-3}$ dust in the tail was produced at $\tau \sim 1 \mathrm{yr}(r \sim 8 \mathrm{au})$, the larger $\beta \sim 10^{-4}$ dust produced at the same time would still have yet to move out of the coma, and the lower brightness of the tail over this syndyne at similar distances from the coma may merely reflect a lower overall dust production rate at $\tau \gtrsim 10 \mathrm{yr}(r \gtrsim 30$ au) than at $\sim 1 \mathrm{yr}$, rather than an absence of larger grains being produced. Detailed dust simulations fitted to the surface brightness of the tail are needed to probe these grains but are beyond the scope of the present analysis.

The spectrum in Figure 2 shows no obvious gas emission lines, so the measured colors represent those of the dust. The dust is redder at shorter wavelengths, with a spectral slope of $16 \% \pm 3 \%$ per $100 \mathrm{~nm}$ between the $\mathrm{HB}$ BC and GC bands $(445-526 \mathrm{~nm})$, dropping to $3 \% \pm 1 \%$ per $100 \mathrm{~nm}$ between $\mathrm{GC}$ and RC $(526-713 \mathrm{~nm})$. The $B-V=0.76 \pm 0.01$, $V-R=0.44 \pm 0.01$, and $R-I=0.39 \pm 0.01$ derived from the spectrum are nearly indistinguishable from the mean colors reported by Jewitt (2015) for a sample of distant long-period comets $(0.78 \pm 0.02,0.47 \pm 0.02$, and $0.42 \pm 0.03$, respectively). The derived F438W-F606W (438-606 nm) slope of $10 \% \pm 1 \%$ per $100 \mathrm{~nm}$ is also substantially redder than the $5.0 \% \pm 0.3 \%$ per $100 \mathrm{~nm}$ found by $\mathrm{Li}$ et al. (2014) for C/ 2013 A1 - a dynamically new comet, unlike C/2021 A1-at similar $r \sim 4$ au.

A'Hearn et al. (1984) introduced the use of the Af $\rho$ parameter as a proxy for the dust production of a comet that requires minimal assumptions about the physical makeup of the dust to compute, unlike the true dust production rate. The $A f \rho$ measured inside a photometric aperture centered on the nucleus is the product of the dust's apparent albedo, $A$; a filling factor, $f$, representing the ratio of scattering cross-section area to aperture area; and the radius, $\rho$, of the aperture and is minimally dependent on $\rho$ for comets with steady-state dust production, where the dust brightness approximately follows a $1 / \rho$ profile.

We measured the Af $\rho$ directly with circular aperture photometry for images from all three epochs. Additionally, although intended for circular apertures, we also estimated the Af $\rho$ over the range of our spectrum in Figure 2 and Table 1 by treating the $10^{\prime \prime} \times 10^{\prime \prime}$ box aperture as equivalent to a $\rho=5$ !. 7 circular aperture, as both would capture an identical flux from a $1 / \rho$ brightness distribution. We measured $A f \rho$ in $V R \sim r^{\prime}$ $(\sim 625 \mathrm{~nm})$ of $1.3 \pm 0.1 \mathrm{~m}$ on 2021 January 13 and HB RC $(\sim 713 \mathrm{~nm})$ of $1.46 \pm 0.06 \mathrm{~m}$ on 2021 March 3 and $1.37 \pm 0.05 \mathrm{~m}$ on 2021 March 20 within effective $\rho \approx 5^{\prime \prime} \approx 15,000 \mathrm{~km}$. Since $A$ is strongly dependent on phase angle $\alpha$, the Af $\rho$ should normally be corrected to the same $\alpha$ before comparison.

We corrected our Af $\rho$ to $\alpha=0^{\circ}$ using the Schleicher-Marcus phase function (Schleicher \& Bair 2011) as an approximation for the true wavelength-dependent phase function of $\mathrm{C} /$ $2021 \mathrm{~A} 1$ and found corresponding $A\left(0^{\circ}\right) f \rho$ of $2.0 \pm 0.2$, $2.2 \pm 0.1$, and $2.1 \pm 0.1 \mathrm{~m}$ in $r^{\prime}$ within $\rho \approx 5^{\prime \prime}$-indistinguishable from constant over our observing period. The Af $\rho$ within a smaller $\rho=2^{\prime \prime} \approx 6000 \mathrm{~km}$ is $\sim 50 \%-70 \%$ larger in images at all epochs and likely better reflects the dust production for the extremely small coma that is $<5^{\prime \prime}$ in radius for comparison with other comets but is noisier and more strongly affected by seeing conditions. These $A\left(0^{\circ}\right) f \rho$ values are all several times smaller than the $\sim 15 \mathrm{~m}$ measured for $\mathrm{C} / 2013 \mathrm{~A} 1$ at similar $r$ (Ye \& 
Hui 2014), which-given the fairly similar $\beta$ of dust observed in the tail-suggests that the dust production of C/2021 A1 is likely lower than that of $\mathrm{C} / 2013 \mathrm{~A} 1$ at this distance, although quantitative constraints on the relative dust albedo and size distributions are required to make a definitive conclusion.

\subsection{Gas Production}

We did not robustly detect any gas emission at either epoch. We used the simple single-component model of Haser (1957) to place approximate upper limits on the production of $\mathrm{CN}, \mathrm{C}_{3}$, and $C_{2}$ with a generous assumed outflow speed of $1 \mathrm{~km} \mathrm{~s}^{-1}$ and adopted the efficiency factors and scale lengths compiled by A'Hearn et al. (1995), except for the formation length of CN. Fray et al. (2005) noted that the breakdown of an unknown parent-possibly dust-is responsible for the short $\mathrm{CN}$ formation lengths of $\sim 10^{4} \mathrm{~km}$ typically observed and assumed for comets near $r \sim 1 \mathrm{au}$, while $\mathrm{HCN}$ photodissociationwhich has a much longer expected scale length of $\sim 8 \times 10^{4} \mathrm{~km} \times(r / 1 \mathrm{au})^{2}$ (Hänni et al. 2020)—should be the principal source of $\mathrm{CN}$ at the $r>3$ au of our observations, and we adopt this latter value as the formation length of $\mathrm{CN}$ in our model. We note that the final production rate estimates increase with increasing formation scale and outflow speed, so an overestimated formation scale and outflow speed provide conservative (i.e., higher) upper limits on gas production.

Since the CN formation scale is larger than the LDT/LMI field of view, we adopt the aperture differencing technique described in Ye et al. (2021) to derive the $\mathrm{CN}$ production rate from the LDT images. This technique compares the measured difference between two apertures against the modeled value and therefore relaxes the need to observe a separate background field. After experimenting with apertures of different radii, we used two annuli with inner/outer radii of $10^{\prime \prime} / 15^{\prime \prime}$ and $45^{\prime \prime} / 60^{\prime \prime}$ to provide the most stringent constraint. The inner annulus purposely excludes the dust coma to avoid needing a continuum correction. Such a correction would require extrapolating the $\mathrm{RC}$ and $\mathrm{BC}$ fluxes to the $\mathrm{CN}$ bandpass, which is inaccurate if the continuum color slope is not constant over the full $\mathrm{CN}-\mathrm{RC}$ range. We also note that $\sim 60^{\prime \prime}$ is the largest usable aperture due to uncorrected stray-light patterns starting $\sim 90^{\prime \prime}$ from the nucleus that reach $\sim 1 \%$ of the background level. Such patterns were not noted in Ye et al. (2021), likely due to their much shorter exposures and much brighter target. We determined the $3 \sigma$ upper limit of the excess flux per wavelength interval within the inner annulus above the surface brightness of the outer annulus to be $8.0 \times 10^{-11} \mathrm{~W} \mathrm{~m}^{-3}$, corresponding to a $3 \sigma \mathrm{CN}$ production limit of $Q(\mathrm{CN})<10^{23.0}$ molec $\mathrm{s}^{-1}$.

We also derived upper limits for $\mathrm{CN}, \mathrm{C}_{3}$, and $\mathrm{C}_{2}$ production on 2021 March 20 from the DBSP spectrum. We first used emcee (Foreman-Mackey et al. 2013) to fit a five-point cubic spline function to our reflectance spectrum. To avoid including the gas emission of interest in this fit, we fitted only points in and near the $\mathrm{HB}$ continuum bands and longward of RC. We approximated the flux uncertainty of all bins as normally distributed and independent, used uniform priors, and verified burn-in completion by visual inspection of the parameter chains. We then measured the residual flux - accounting for both the flux and continuum uncertainty-through the $\mathrm{HB} \mathrm{CN}$, $\mathrm{C}_{3}$, and $\mathrm{C}_{2}$ and bandpasses, scaled to the full-band $\mathrm{CN}$ $(\Delta v=0), \mathrm{C}_{3}$ (Swings), and $\mathrm{C}_{2}(\Delta v=0)$ fluxes following Farnham et al. (2000), then converted the $3 \sigma$ upper bounds to column densities and subsequently production rates by the simple Haser (1957) models described earlier. This procedure yielded $3 \sigma$ upper bounds of $Q(\mathrm{CN})<10^{23.3}, Q\left(\mathrm{C}_{3}\right)<10^{22.4}$, and $Q\left(\mathrm{C}_{2}\right)<10^{23.0}$ molec s$^{-1}$, which are roughly equally stringent for typical cometary abundances (A'Hearn et al. 1995).

While no gas emission was detected, we can compare the $Q$ (CN) upper limits with the $A\left(0^{\circ}\right) f \rho=2.9 \pm 0.1 \mathrm{~m}$ measured by LDT in RC within $\rho=2^{\prime \prime}$ and define a ratio $\mathrm{CN} / 5$ $A f \rho \equiv Q(\mathrm{CN}) /\left(A\left(0^{\circ}\right) f \rho\right)$ for which we find $\mathrm{CN} / A f \rho<10^{22.5}$ molec s $\mathrm{s}^{-1} \mathrm{~m}^{-1}$. This bound is several times smaller than the smallest CN/Af $\rho$ measured by A'Hearn et al. (1995) after an approximate correction of the latter to a $0^{\circ}$ phase angle, although the vast majority of measurements were made at much smaller $r$. Knight \& Schleicher (2014) reported a detection of $\mathrm{CN} / A f \rho=10^{24.1 \pm 0.2}$ molec s$^{-1} \mathrm{~m}^{-1}$ for the dynamically new sungrazing comet $\mathrm{C} / 2012 \mathrm{~S} 1$ (ISON) at $r=4.6$ au preperihelion. Similarly, Rauer et al. (1997) found $\mathrm{CN} / A f \rho=10^{23.5} \pm 0.5$ molec s${ }^{-1} \mathrm{~m}^{-1}$ for the very large $\mathrm{C} / 1995 \mathrm{O} 1$ (Hale-Bopp) at $r=4.6 \mathrm{au}$. No measurements of $Q(\mathrm{CN})$ were made of $\mathrm{C} /$ 2013 A1 until it had already reached $r=2.4$ au, where Opitom et al. (2016) found $\mathrm{CN} / A f \rho=10^{24.0 \pm 0.1}$ molec s$^{-1} \mathrm{~m}^{-1}$.

The interpretation and implications of the low $\mathrm{CN} / A f \rho$ observed for $\mathrm{C} / 2021 \mathrm{~A} 1$ are unclear without a bigger sample of measurements for comparable long-period comets observed at similar $r$. The absence of the non-HCN parent of $\mathrm{CN}$ at $r>3$ au is likely at least partially responsible. We also speculate that $\mathrm{HCN}$-which sublimates at a lower temperature than $\mathrm{H}_{2} \mathrm{O}$ - may be somewhat depleted by thermal fractionation from the outer layers of $\mathrm{H}_{2} \mathrm{O}$ ice on the comet, the latter of which may already be weakly sublimating and driving dust production at $r \sim 5 \mathrm{au}$ (Meech \& Svoren 2004). Another possibility is that our $A f \rho$ apertures are sampling an abundance of old, slow-moving grains in the coma and the portion of the tail behind the coma, thus leading the measured Af $\rho$ to be more reflective of the comet's accumulated dust production history rather than its ongoing dust production. In all of these cases, we anticipate that $\mathrm{CN} / A f \rho$ will rise to more typical values as the non-HCN source begins to contribute, more efficient $\mathrm{H}_{2} \mathrm{O}$ sublimation clears any fractionated layer, old dust grains leave the coma, and changing viewing geometry moves the tail out from behind the coma as the comet approaches the Sun.

\section{Dust Dynamics}

The $b$-plane of a close encounter of a minor to a major planetary body-in our case, C/2021 A1 to Venus-is defined as the plane containing the center of mass of the major body (Venus) that is normal to the inbound asymptotic approach velocity $v_{\infty} \equiv v_{\infty} \hat{\boldsymbol{v}}_{\infty}$ of the minor body (C/2021 A1) and serves as a useful tool for describing and analyzing such close encounters (Kizner 1961). We adopted the commonly used formalism of Valsecchi et al. (2003), which labels the plane by two coordinates, $\xi$ and $\zeta$, associated with the orthonormal unit vectors $\hat{\xi}$ and $\hat{\zeta}$, with Venus at the origin, and with $\hat{\zeta} \times \hat{\xi}=\hat{\boldsymbol{v}}_{\infty}$ rotated such that the projected heliocentric velocity of Venus falls along $-\zeta$.

Figure 3 shows the $b$-plane crossing points for dust of several representative $\beta$ and ejection ages $\tau=\tau_{0}$ defined from the $b$-plane crossing time of the nucleus $T_{0}$-effectively, the time of its closest approach to Venus (2021 December 18 02:08 UT \pm 3 minutes; JPL orbit solution 9). While each dust grain will technically have a different $b$-plane, their relative 


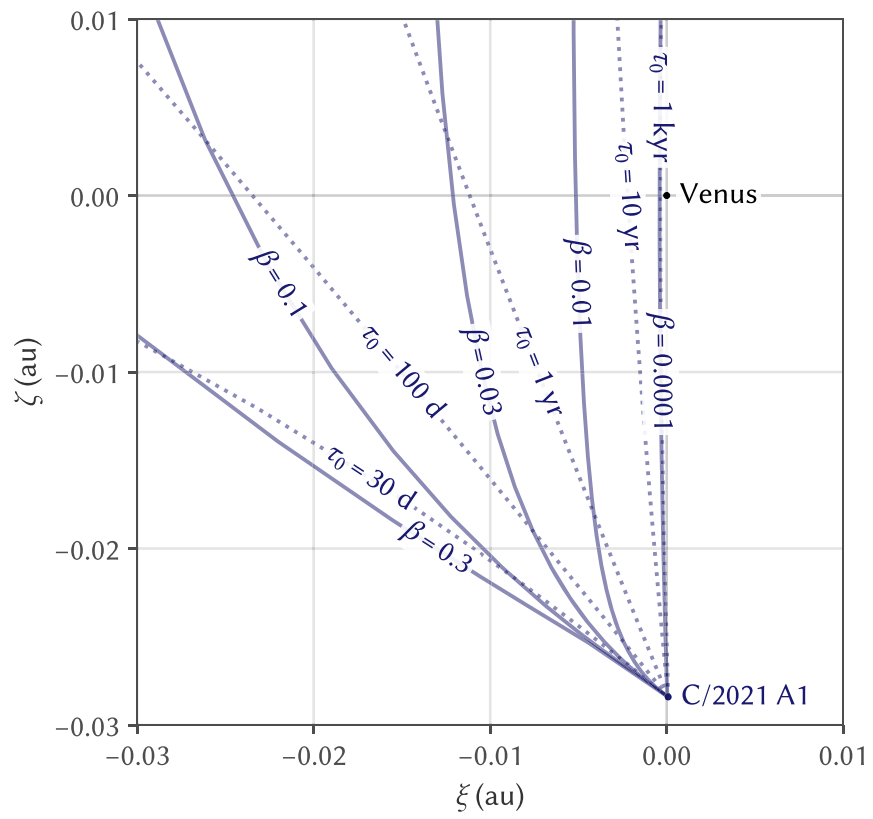

Figure 3. Syndynes (solid curves) and synchrones (dotted curves) of C/ 2021 A1 projected onto the $b$-plane of its encounter with Venus, illustrating the closest approach points of $v_{d}=0$ dust. Venus falls $\sim 50,000 \mathrm{~km}$ from the edge of this dust fan, so a small but nonzero $v_{d}$ is required for any dust to reach Venus. Note that the labeled dots indicating the positions of Venus and the nucleus of $\mathrm{C} / 2021 \mathrm{~A} 1$ are both much larger than the respective objects, for visibility.

velocity with respect to each other and the nucleus is much smaller than their speed with respect to Venus, which ensures that all encounter trajectories are nearly parallel and thus enables the $\xi$ and $\zeta$ of their crossing points to be usefully compared together in this manner.

Additionally, the asymptotic approach speed of $v_{\infty}=78$ $\mathrm{km} \mathrm{s}^{-1}$ of $\mathrm{C} / 2021 \mathrm{~A} 1$ to Venus is much faster than the escape speed of $10.4 \mathrm{~km} \mathrm{~s}^{-1}$ from the surface of Venus, so gravitational deflection of both the comet nucleus and all dust grains moving with a similar velocity by Venus will be negligible. In this case, the $\xi$ and $\zeta$ where the nucleus and all associated dust grains cross the $b$-plane represents the spatial position of each respective object's closest approach to the planet. All crossing points $\xi^{2}+\zeta^{2} \lesssim \tilde{R}_{\text {ven }}^{2}$ therefore represent impact on the planet, where we have adopted $\tilde{R}_{\mathrm{ven}}=6200 \mathrm{~km}$ as the solid radius of Venus, plus an $\sim 100 \mathrm{~km}$ buffer as a rough estimate for the portion of the atmosphere within which grazing meteors may fully ablate, plus an additional $\sim 50 \mathrm{~km}$ from gravitational focusing of $v_{\infty}=78 \mathrm{~km} \mathrm{~s}^{-1}$ particles by Venus.

As Venus falls just outside the $v_{d}=0$ dust fan by the MOID of $\sim 50,000 \mathrm{~km}$, the planet will not encounter an abundance of low- $v_{d}$ dust grains when it crosses the comet's orbital plane and will, instead, only intercept the higher- $v_{d}$ grains that drift to it away from the main fan. To evaluate the plausibility of dust grains reaching Venus, we define a transformation that projects the initial ejection velocity $\boldsymbol{v}_{\boldsymbol{d}}$ for each dust grain of $\beta$ and $\tau_{0}$ onto its corresponding $b$-plane crossing point $(\xi, \zeta)\left(\beta, \tau_{0}, \boldsymbol{v}_{\boldsymbol{d}}\right)$. This transformation is nearly linear under the linear encounter approximation described above when considering only $\left(\xi^{2}+\zeta^{2}\right)^{1 / 2} \ll 0.7$ au (i.e., Venus's orbit radius) and can therefore be described by Equation (1):

$$
\begin{aligned}
(\xi, \zeta)\left(\beta, \tau_{0}, \boldsymbol{v}_{\boldsymbol{d}}\right) & =(\xi, \zeta)\left(\beta, \tau_{0}, 0\right)+\boldsymbol{X}_{\beta, \tau_{0}} \boldsymbol{v}_{\boldsymbol{d}}, \\
\boldsymbol{X}_{\beta, \tau_{0}} & \equiv \frac{\partial(\xi, \zeta)}{\partial \boldsymbol{v}_{\boldsymbol{d}}}\left(\beta, \tau_{0}, 0\right) .
\end{aligned}
$$

Here $\boldsymbol{X}_{\beta, \tau_{0}}$ is a $2 \times 3$ Jacobian matrix relating displacements in $\boldsymbol{v}_{\boldsymbol{d}}$ to displacements in $\xi, \zeta$. An isotropic shell of dust ejected at equal speed $v_{d}$ with a given $\beta$ and $\tau_{0}$ therefore projects onto a footprint ellipse in the $b$-plane that is centered on the associated $v_{d}=0$ crossing point, with the semiaxes of the ellipse given by $v_{d}$ multiplied by the singular values of $\boldsymbol{X}_{\beta, \tau_{0}}$.

For each $\beta$ and $\tau_{0}$, we compute $(\xi, \zeta)\left(\beta, \tau_{0}, 0\right)$ by two-body Keplerian trajectories from the comet's heliocentric osculating orbit at $T_{0}$ to optimize computing resource usage and subsequently calculate $\boldsymbol{X}_{\beta, \tau_{0}}$ by finite differences. As $a_{\text {in }} \approx 1900 \mathrm{au}$, this approximation is only valid out to $\sim 1000$ au before the osculating orbit and the true trajectory substantially diverge. Planetary perturbations will minimally alter the relative trajectory of the nucleus and dust grains, since all relevant trajectories are tightly clustered together compared to interplanetary distances and thus experience nearly the same perturbations. We then follow Farnocchia et al. (2014) and use Lagrange multipliers to determine the minimum speed $v_{d}=v_{0}$ with which a dust grain with a given $\beta$ and $\tau_{0}$ must be ejected in order to reach Venus, representing a first criterion for any dust grains with a particular set of parameters to be become meteors. The first plot of Figure 4 shows that the required $v_{0}$ generally decreases with increasing $\tau_{0}$ but with each $\beta$ having an optimum $\tau_{0}$ where $v_{0}$ is minimized, corresponding to the $\beta$ and $\tau_{0}$ combinations with $(\xi, \zeta)\left(\beta, \tau_{0}, 0\right)$ near the origin. These results and the associated approximations were validated by spot checking with full $n$-body simulations that include the gravity of the Sun and eight major planets, which indicate that the computed $v_{0}$ are generally accurate to within $\sim 10 \%$ for $r\left(\tau_{0}\right) \lesssim 30 \mathrm{au}$ and within a factor of about 2 for $r\left(\tau_{0}\right) \lesssim 1000 \mathrm{au}$.

While grains ejected at $v_{d}>v_{0}$ in certain directions can reach Venus, only a small fraction of ejected grains will actually do so. This fraction depends on the size of the footprint ellipse, with a larger footprint generally translating to a smaller fraction of dust reaching Venus once $v_{d}>v_{0}$ is met. We consider a meteor production efficiency $f_{\mathrm{V}}\left(v_{0}\right)$, defined as the ratio of $\pi \tilde{R}_{\mathrm{ven}}^{2}$ to the area of the footprint ellipse for $v_{d}=v_{0}$, which very roughly corresponds to the fraction of dust ejected isotropically at $v_{d} \sim v_{0}$ that will impact Venus. The second plot of Figure 4 shows that $f_{\mathrm{V}}\left(v_{0}\right)$ is a maximum of $\sim 0.03 \%$ for this encounter for dust grains of $\beta \sim 10^{-3}-10^{-4}$ ejected at $r\left(\tau_{0}\right) \sim 70-300 \mathrm{au}$ with $v_{d} \sim v_{0} \sim 10^{-1} \mathrm{~m} \mathrm{~s}^{-1}$, comparable to the escape speed from the surface of a kilometer-scale nucleus. Larger and older grains will generally have even lower $v_{0}$ but correspond to larger $b$-plane footprints and thus lower $f_{\mathrm{V}}\left(v_{0}\right)$.

The last plot of Figure 4 shows the impact time $t_{0}$ for $v_{d}=v_{0}$ dust grains. Meteors associated with distant activity $\left(\beta \lesssim 10^{-2.5}, \quad r\left(\tau_{0}\right) \gtrsim 30 \quad\right.$ au $)$-where efficiency $f_{\mathrm{V}}\left(v_{0}\right)>0.01 \%$ - will largely be concentrated to within $\sim 1 \mathrm{hr}$ of 2021 December 19 21:00 UT, when Venus passes through its MOID point with the comet's heliocentric orbit. Any meteors resulting from more recent dust production may begin as soon as $\sim 1.5$ days earlier near $T_{0}$.

However, no meteors will occur at any time if the comet does not actually produce any dust of $v_{d} \gtrsim v_{0}$ on its approach. Dust produced since the comet's earliest observations at $r=7.5$ au requires a very high $v_{d}>v_{0}>100 \mathrm{~m} \mathrm{~s}^{-1}$ to reach 


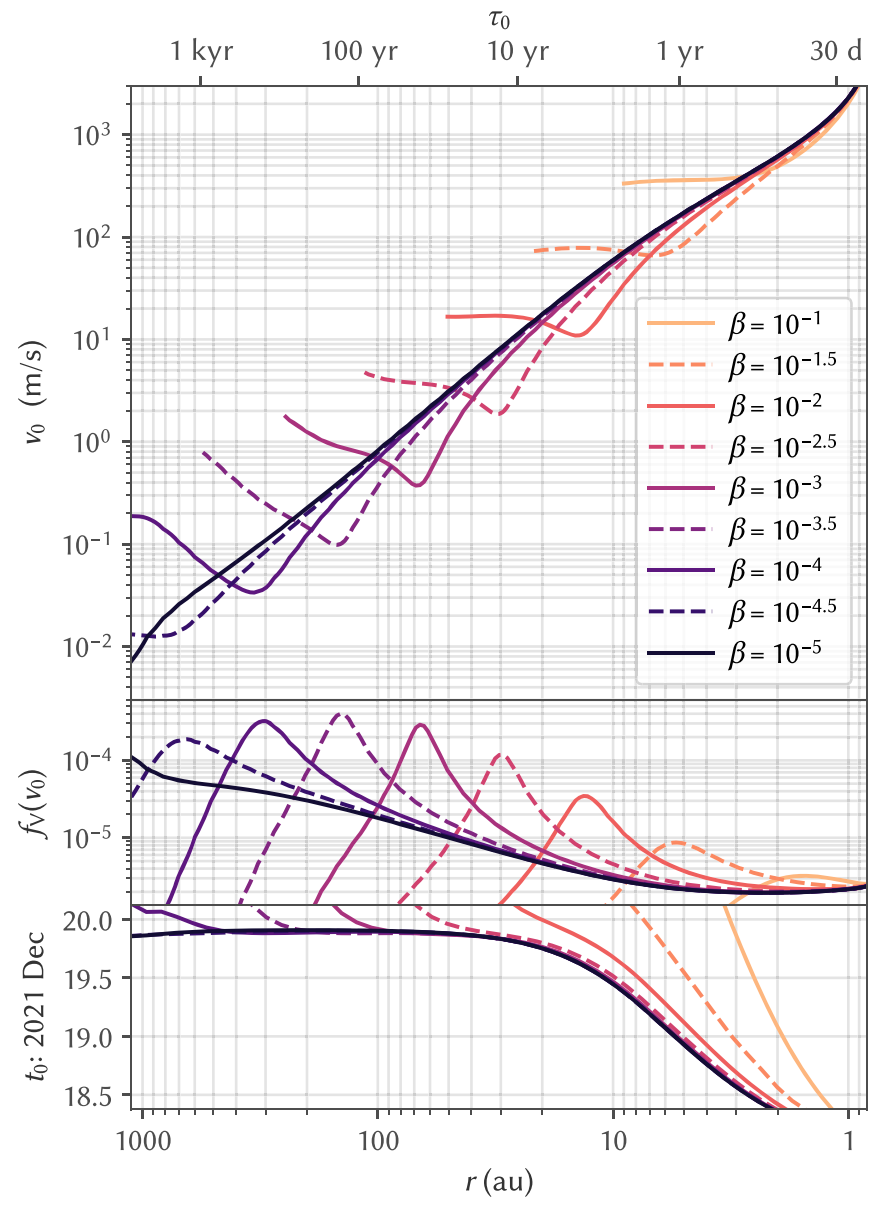

Figure 4. Minimum $v_{d}$ for dust of various $\beta$ ejected at various $r$ to reach Venus $\left(v_{0}\right)$, the approximate fraction of dust isotropically ejected with $v_{d} \sim v_{0}$ reaching Venus $\left(f_{\mathrm{V}}\left(v_{0}\right)\right)$, and the time $t_{0}$ at which they do so.

Venus. A $\beta=10^{-2}$ dust grain-near the average observed from $\mathrm{C} / 2021 \mathrm{~A} 1$ - ejected at $v_{d} \sim 100 \mathrm{~m} \mathrm{~s}^{-1}$ in the sunward direction while at $r \sim 5-10$ au would experience radiation pressure acceleration relative to the nucleus of $\sim 10^{-6} \mathrm{~m} \mathrm{~s}^{-2}$ in the antisunward direction. The grain would not be turned around and pushed into a tail for $>1 \mathrm{yr}$ while $\gtrsim 10^{6} \mathrm{~km}$ from the nucleus. The observed dust coma, however, is 2 orders of magnitude smaller yet already folded into a tail, so we do not observe dust grains ejected near this speed.

Aside from the cases of an extremely large nucleus (Jewitt \& Matthews 1999) and near-Sun comets well within the orbit of Venus (Jones et al. 2018) - neither of which apply to C/ $2021 \mathrm{~A} 1$ - the dust of the observed $\beta \lesssim 10^{-2}$ and the needed $v_{d}>100 \mathrm{~m} \mathrm{~s}^{-1}$ are only produced by explosive outbursts where the gas is of sufficient density to accelerate dust to speeds comparable to the outflow speed of up to $\sim 1 \mathrm{~km} \mathrm{~s}^{-1}$. Such high-speed outbursts and the distinctive dust shell morphology they produce are occasionally observed from Jupiter family comets like 15P/Finlay at $r \sim 1$ au (Ye et al. 2015) and 17P/ Holmes at $2.4 \mathrm{au}$ (Russo et al. 2008), as well as from active centaurs like 29P/Schwassmann-Wachmann (Trigo-Rodríguez et al. 2008) and 174P/Echeclus (Rousselot 2008) at $r>5$ au. One such outburst was also observed from the long-period comet C/2012 X1 (LINEAR) at 2.7 au (Miles 2013). A dust shell produced by such an outburst from $\mathrm{C} / 2021 \mathrm{~A} 1$ expanding at $v_{d} \gtrsim 0.5 \mathrm{~km} \mathrm{~s}^{-1}$ can reach Venus if the outburst occurs before the comet reaches $r \approx 2 \mathrm{au}$ in 2021 September.
However, given the exceptional rarity of high-speed dust outbursts from long-period comets, such an event appears highly unlikely for C/2021 A1. Therefore, neither the comet's observed past activity nor its future activity are likely to yield any meteors on Venus, and the most likely source for meteors on Venus becomes dust-producing activity on the comet from before it was first observed.

Additionally, the comet's large $a_{\text {in }} \approx 1900$ au implies that perihelion activity from previous apparitions will not substantially affect the meteoroid flux at Venus. Even without nongravitational forces (i.e., $\beta \rightarrow 0$ ), particles separated from the nucleus with a modest $v_{d}=1 \mathrm{~ms}^{-1}$ at the previous perihelion will be widely spaced along the orbit, preceding or trailing the nucleus by up to $\sim 30 \mathrm{kyr}$ in the current apparition. We therefore expect no substantial enhancement in the meteoroid flux near the nucleus from activity on previous apparitions. Any meteor activity observed on Venus from C/ 2021 A1 would thus most likely originate from distant activity by the comet from earlier in the present apparition.

\subsection{Distant Dust Production}

Preobservational distant activity on C/2013 A1 at $r>10$ au was inferred from the meteor shower it produced on Mars. In that encounter, Mars lay on the $b$-plane at the intersection of the $\beta=1.43 \times 10^{-4}$ syndyne and the $r\left(\tau_{0}\right)=22.5$ au synchrone and thus efficiently intercepted dust grains produced with these and similar properties (Farnocchia et al. 2014). While the earliest analyses by Moorhead et al. (2014) and Vaubaillon et al. (2014) suggested that an abundance of younger, highvelocity grains could potentially dominate the meteoroid flux at Mars, they required comet behavior that was ultimately inconsistent with the observed dust production of the comet. Ye \& Hui (2014), Tricarico et al. (2014), and Li et al. (2014) directly detected and thus constrained the quantity and velocity of $\beta \lesssim 10^{-2}$ grains produced by the comet, showing that the small and recently produced grains were far too slow to reach Mars. Kelley et al. (2014) likewise simulated dust production starting from $r=13 \mathrm{au}$, showing that there would be negligible meteor activity from dust produced within this distance.

The remaining possibility of a substantial level of large grains of $\beta \sim 10^{-3}-10^{-4}$, or $a_{d} \sim 1-10 \mathrm{~mm}$, being produced at $r>10$ au had not been seriously considered prior to the encounter, so the MAVEN IUVS detection of a temporary metallic vapor layer lasting $\sim 2$ days with a strength consistent with $\sim 10^{4} \mathrm{~kg}$ of ablated meteoroids from the comet (Schneider et al. 2015)_far in excess of the layer's quiescent level (Crismani et al. 2017)—was largely unexpected. Those observations were supplemented by detections of an apparent influx of ions-particularly metallic ions likely of meteoritic origin - into the planet's ionosphere by instruments on board MAVEN (Benna et al. 2015), the Mars Reconnaissance Orbiter (Restano et al. 2015), and Mars Express (Sánchez-Cano et al. 2020).

We note that Schneider et al. (2015) found the high $\sim 120 \mathrm{~km}$ altitude of the metallic vapor layer-and thus of meteor ablation-to be consistent with predominantly $a_{d} \sim 1-100 \mu \mathrm{m}$ meteoroids of $\rho_{d}<1 \mathrm{~g} \mathrm{~cm}^{-3}$, which they suggested implies that intercepted grains were actually recently ejected from the nucleus at very high $v_{d}$, rather than the expected 1-10 mm grains from $r>10 \mathrm{au}$. However, $\sim 10^{4} \mathrm{~kg}$ of grains of $a_{d} \sim 1-100 \mu \mathrm{m}$ with a typical $\rho_{d} \sim 0.3-1 \mathrm{~g} \mathrm{~cm}^{-3}$ and $\sim 4 \%$ albedo spread over the cross-sectional area of Mars 
would have had a readily observable $V$-band surface brightness of 21-27 mag arcsec ${ }^{-2}$, while telescopic observations of the comet during the encounter were consistent with pre-encounter observations and revealed no such high- $v_{d}$ dust (Li et al. 2016). Therefore, the intercepted grains were most likely the expected 1-10 mm grains from $r>10 \mathrm{au}$, and we speculate that the high ablation altitude may instead reflect a weaker grain structure that is more prone to fragmentation than exhibited by the much older meteoroids in Earth-crossing streams-which traditional meteor models are largely based on-as such fragile grains in these multiapparition streams may have already broken down prior to reaching Earth.

Such distant dust activity may not be unusual. More recently, the large Oort cloud comet C/2017 K2 (PANSTARRS) was directly observed to be producing millimeter-sized grains at $r>20$ au (Hui et al. 2017). This activity appears consistent with surface sublimation of CO (Meech et al. 2017; Jewitt et al.

2019; Yang et al. 2021), a process that can be thermally efficient out to $r \sim 120$ au (Meech \& Svoren 2004). Jewitt et al. (2021) concluded from monitoring of the comet's dust morphology that the observed dust activity was underway out to at least $r \approx 35 \mathrm{au}$, finding a dust production rate following

$$
\dot{m} \approx \dot{m}_{10} \times(10 \mathrm{au} / r)^{2}
$$

with $\dot{m}_{10} \sim 1000 \mathrm{~kg} \mathrm{~s}^{-1}$. They also derived a model for the mean dust ejection speed

$$
\left\langle v_{d}\right\rangle \approx v_{10} \times \beta^{0.5} \times(10 \mathrm{au} / r)
$$

and measured $v_{10} \approx 170 \mathrm{~m} \mathrm{~s}^{-1}$ for $\mathrm{C} / 2017 \mathrm{~K} 2$, a value consistent with an $R_{\text {nuc }} \approx 6 \mathrm{~km}$ radius nucleus covered in $\mathrm{CO}$ ice, following a theoretical $v_{10} \propto R_{\text {nuc }}^{1 / 2}$ relation.

Object C/2021 A1 is intrinsically much fainter than $C /$ 2017 K2 and thus likely to be much smaller, have a much less volatile surface, or both. The dust-contaminated magnitudes reported in the earliest prediscovery observations (Leonard et al. 2021a) set only a very weak upper bound of $R_{\text {nuc }} \lesssim 7 \mathrm{~km}$, assuming a typical $\sim 4 \%$ albedo. Additionally, the barycentric $a_{\text {in }} \approx 1900$ au suggests that the comet previously survived a perihelion passage of a similar $q \approx 0.6 \mathrm{au}$ to its present apparition at least once before (Królikowska \& Dybczyński 2017), while dynamically new Oort cloud comets of $R_{\text {nuc }} \lesssim 0.5 \mathrm{~km}$ frequently disintegrate at comparable or larger $r$ (e.g., Farnham et al. 2001; Li \& Jewitt 2015; Combi et al. 2019). Although we lack a rigorous statistical analysis of survival rate by nucleus size, the survival of $\mathrm{C} / 2021 \mathrm{~A} 1$ crudely suggests $R_{\text {nuc }} \gtrsim 0.5 \mathrm{~km}$, and we adopt $R_{\text {nuc }} \approx 1 \mathrm{~km}$ as the expected value, reflecting both the lower limit and the bottom-heavy size distribution of comet nuclei (Boe et al. 2019). This assumed size is additionally comparable to the lower bound of $R_{\text {nuc }}>0.9 \mathrm{~km}$ obtained by Schleicher et al. (2002) for $\mathrm{C} / 2000 \mathrm{WM}_{1}$ (LINEAR), a comet with a very similar $a_{\text {in }} \approx 1900$ au and $q \approx 0.6$ au to C/2021 A1, as well as a similar brightness at $r \sim 5$ au preperihelion.

A nucleus of $R_{\text {nuc }} \approx 1 \mathrm{~km}$ gives $v_{10} \approx 70 \mathrm{~m} \mathrm{~s}^{-1}$ under Jewitt et al.'s (2021) CO surface sublimation model. This $v_{10}$ implies that a grain of any $\beta$ ejected at any $r$ in the sunward direction will be turned around by radiation pressure at a distance of $d_{\mathrm{ta}} \sim 80,000 \mathrm{~km}$ away from the nucleus, setting a minimum size for the coma. Figure 1, however, shows a much smaller coma with a sunward edge only $\sim 3^{\prime \prime}$ from the nucleus in the first epoch, which translates to $d_{\mathrm{ta}} \sim 2000 \mathrm{~km}$ after correcting for $\alpha \approx 11^{\circ}$ by approximating the coma as a paraboloid. The

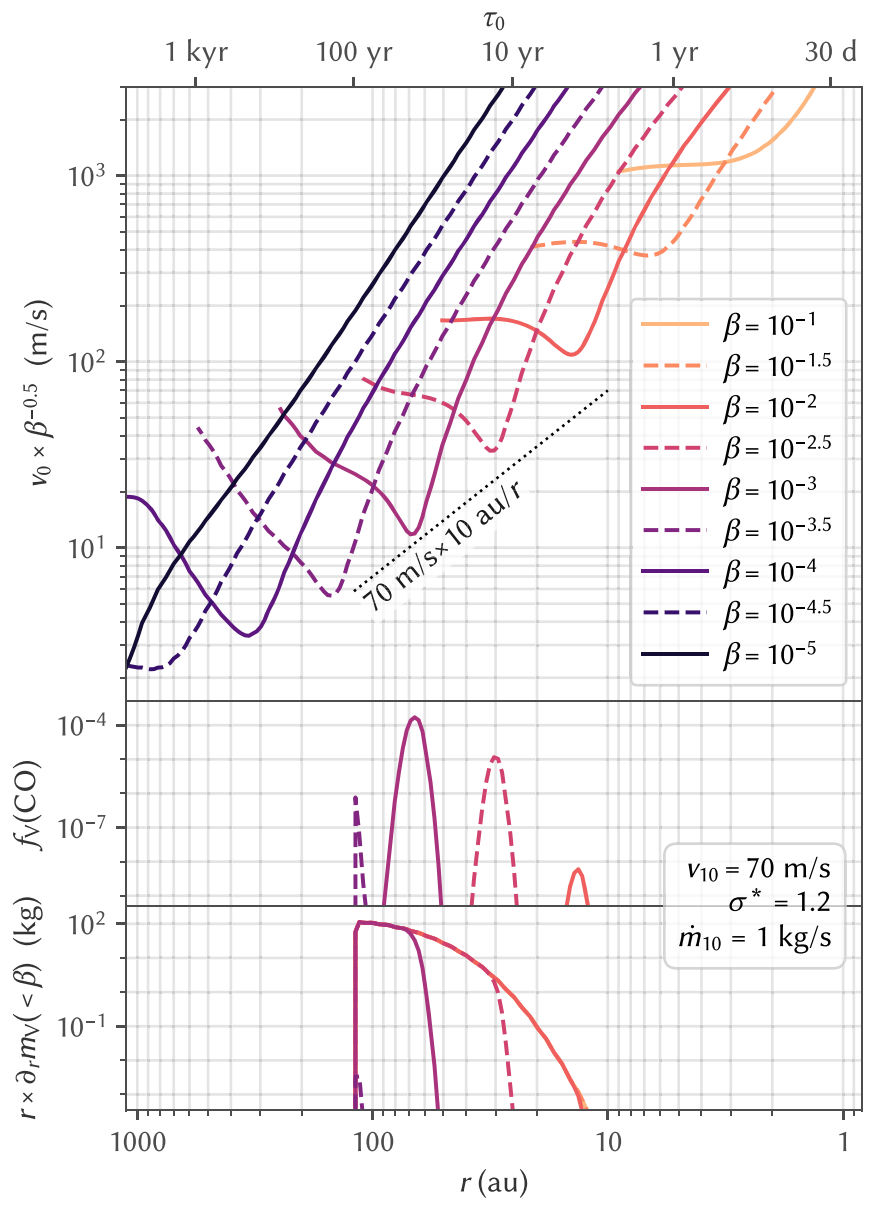

Figure 5. The $v_{0}$ from Figure 4 scaled by $\beta^{-0.5}$ relative to $v_{10}=70 \mathrm{~m} \mathrm{~s}^{-1}$ for a $2 \mathrm{~km}$ diameter nucleus with $\mathrm{CO}$ surface sublimation, the fraction $f_{\mathrm{V}}(\mathrm{CO})$ of such dust intercepted by Venus, and the intercepted mass $m_{\mathrm{V}}$ below the indicated $\beta$ produced per unit interval of $\ln r$-denoted by $r \times \partial_{r} m_{\mathrm{V}}(<\beta)$ serving as a rough approximation for the intercepted mass produced near $r$.

small $d_{\mathrm{ta}} \sim 2000 \mathrm{~km}$ corresponds to a much lower $v_{10} \sim 10$ $\mathrm{m} \mathrm{s}^{-1}$, so the nucleus evidently does not contain the large quantities of actively sublimating $\mathrm{CO}$ as that of $\mathrm{C} / 2017 \mathrm{~K} 2$ appears to have. For comparison, C/2013 A1 was observed to have $v_{10} \sim 20 \mathrm{~m} \mathrm{~s}^{-1}$ at similar $r$ (Ye \& Hui 2014). We note, however, that $v_{10}$ may have been higher in the past if the $r \sim 5$ au activity was being driven by a less volatile substance, like $\mathrm{CO}_{2}$ or $\mathrm{H}_{2} \mathrm{O}$, or if the surface only became depleted of $\mathrm{CO}$ earlier during its present approach to the Sun. The latter could result from seasonal effects if a portion of the nucleus remained shaded through the outbound leg of its previous apparition and was thus still freshly resurfaced from perihelion until efficient $\mathrm{CO}$ sublimation began at $r \sim 120$ au. We therefore regard $v_{10} \approx 70 \mathrm{~m} \mathrm{~s}^{-1}$ as a plausible, albeit unlikely, upper limit for dust produced by quiescent surface sublimation.

The first plot of Figure 5 modifies Figure 4 by scaling the calculated $v_{0}$ by a factor $\beta^{-0.5}$ to simultaneously compare the necessary $v_{0}$ for dust grains of different sizes, since the quantity $v_{d} \times \beta^{-0.5}$ is approximately independent of $\beta$ under the model in Equation (3). The plot shows that the $v_{10} \approx 70 \mathrm{~m} \mathrm{~s}^{-1}$ of a $\mathrm{CO}$-covered surface at $r<120 \mathrm{au}$ is nearly the minimum required for dust of any size released by the comet at any point on its current apparition to have the needed $v_{d} \geqslant v_{0}$ to reach Venus. The second plot shows the corresponding $f_{\mathrm{V}}$ for the footprint ellipse set by $v_{10}=70 \mathrm{~m} \mathrm{~s}^{-1}$, which is a rough 


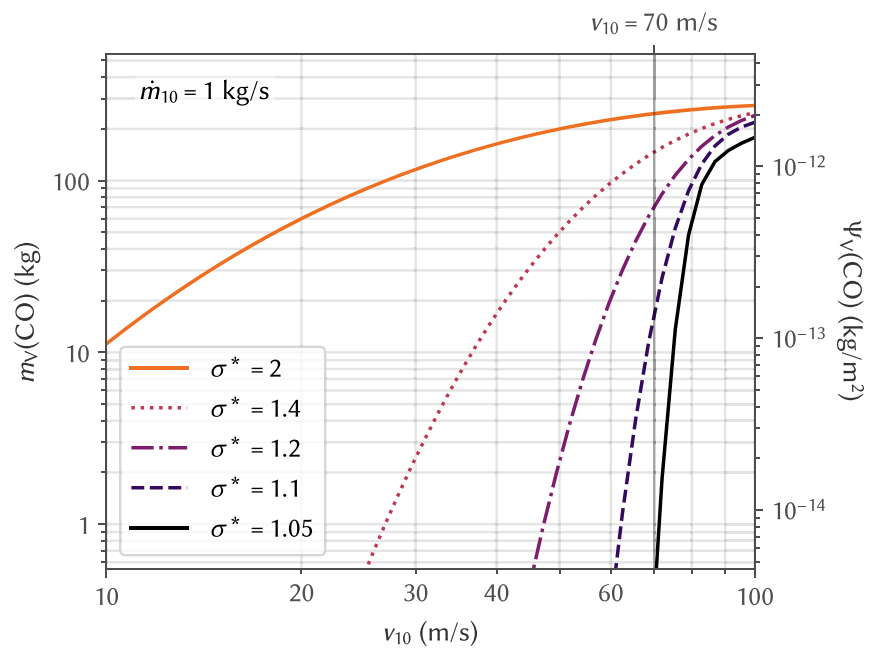

Figure 6. Total mass $m_{\mathrm{V}}(\mathrm{CO})$ and mass flux density $\Psi_{\mathrm{V}}(\mathrm{CO})$ intercepted by Venus as functions of typical normalized dust ejection speed $v_{10}$ and geometric dust speed dispersion $\sigma^{*}$ for isotropic dust production $\dot{m} \propto r^{-2}$ and $\dot{m}_{10} \equiv \dot{m}(10 \mathrm{au})=1 \mathrm{~kg} \mathrm{~s}^{-1}$ from the CO sublimation limit $r=120 \mathrm{au}$ to $10 \mathrm{au}$.

approximation of the dust ejected under this model of a given $\tau_{0}$ and $\beta$ that impact Venus. We also consider $v_{d} \times \beta^{-0.5}$ to be lognormally distributed about the nominal value, with a geometric standard deviation $\sigma^{*}$, and select a plausible $\sigma^{*}=1.2$ for this figure. The true $\sigma^{*}$ is affected by many factors, including the distribution of particle drag coefficients and nucleus nonuniformity, and a precise computation is beyond the scope of this analysis. We also consider a dust mass production rate of $\dot{m}_{10}=1 \mathrm{~kg} \mathrm{~s}^{-1}$ with Equation (2) for the third plot, which shows the mass $m_{\mathrm{V}}(<\beta)$ of all dust grains larger than the indicated $\beta$ reaching Venus under this model produced per unit interval of $\ln r$, denoted by $r \times \partial_{r} m_{\mathrm{V}}(<\beta)$. The plot shows that these meteoroids intercepted by Venus will be principally by $\beta \sim 10^{-3}$ grains produced near $r \sim 100 \mathrm{au}$ around the maximum $r$, where efficient $\mathrm{CO}$ sublimation is possible.

As $v_{10}=70 \mathrm{~m} \mathrm{~s}^{-1}$ is very near the threshold speed for any dust to reach Venus, the cumulative mass of meteoroids intercepted by Venus $m_{\mathrm{V}}(\mathrm{CO})$ under this $\mathrm{CO}$ sublimation model is extremely sensitive to the precise values of $v_{10}$ and $\sigma^{*}$. Figure 6 plots the intercepted mass $m_{\mathrm{V}}(\mathrm{CO})$ and corresponding mass flux $m_{\mathrm{V}}(\mathrm{CO})$ for a variety of $v_{10}$ and $\sigma^{*}$ for the adopted $\dot{m}_{10}=1 \mathrm{~kg} \mathrm{~s}^{-1}$. A substantial meteoroid flux at Venus occurs only for high $v_{10} \gtrsim 70 \mathrm{~m} \mathrm{~s}^{-1}$ or high $\sigma^{*} \gtrsim 1.2$, which will produce $m_{\mathrm{V}}(\mathrm{CO}) \sim 10^{2} \mathrm{~kg}$ of meteors on Venus. We note that the model treats dust as noninteracting particles that can be linearly superposed, so the results may be scaled to any $\dot{m}_{10}$ by $m_{\mathrm{V}}(\mathrm{CO}) \propto \dot{m}_{10}$. However, if the $v_{10} \sim 10 \mathrm{~m} \mathrm{~s}^{-1}$ observed near $r \sim 5$ au held throughout its approach, the comet would produce negligible meteor activity on Venus without an exceptionally high $\sigma^{*} \gtrsim 2$, where a substantial fraction of dust is ejected at several times the nominal speed. In contrast, an equivalent model for C/2013 A1's encounter with Marswhere Mars actually passed within the $v_{d}=0$ dust fan-gives the observed $\sim 10^{4} \mathrm{~kg}$ of meteors for the adopted $\dot{m}_{10} \sim 1$ $\mathrm{kg} \mathrm{s}^{-1}$ at all reasonable $v_{10}$ and $\sigma^{*}$, indicating that $\mathrm{CO}$ surface sublimation adequately explains the meteor activity on Mars from C/2013 A1.

Distant activity may also be driven by mechanisms other than the quiescent surface sublimation we have modeled. For example, Sekanina \& Kracht (2014) noted that C/2012 S1 was trailed by a stream of dust extending several degrees behind the nucleus, which syndyne-synchrone analysis indicates was comprised of $\beta \lesssim 10^{-3}$ grains ejected at $r \gtrsim 10 \mathrm{au}$, and suggested based on the sublimation behavior that it may have contained pebbles of $\beta \sim 10^{-4}$ from as far as $r \sim 100 \mathrm{au}$ produced by annealing of amorphous ice. DiSanti et al. (2016) and Feldman et al. (2018) found that the comet had a low $Q(\mathrm{CO}) / Q\left(\mathrm{H}_{2} \mathrm{O}\right) \sim 1.5 \%$ near $r \sim 1$ au, which suggests that $\mathrm{CO}$ surface sublimation was unlikely to have been responsible for the distant activity, unless the surface abundance of $\mathrm{CO}$ was considerably higher earlier.

Extremely distant activity has also been inferred from the trajectories of fragmented comets. The comet pair C/1988 F1 (Levy) and C/1988 J1 (Holt) were discovered following nearly the same orbit spaced only 76 days apart-too close for the progenitor to have split on its previous apparition, yet too far to have split after entering the planetary region on its current apparition (Marsden 1988). Simulations by Sekanina \& Kracht (2016) suggest that the pair likely separated at $\sim 1 \mathrm{~m} \mathrm{~s}^{-1}$ centuries earlier at $r \sim 300-900 \mathrm{au}$, already on its approach to perihelion. Likewise, the arrival clustering of the Kreutz sungrazing comet family appears to require its members to split decades before perihelion at $r \gtrsim 50$ au and $\sim 10 \mathrm{~m} \mathrm{~s}^{-1}$ (Sekanina \& Chodas 2004).

The physical mechanisms behind the distant fragmentation events are unclear, and no obvious large fragments or companions to $\mathrm{C} / 2021 \mathrm{~A} 1$ had been discovered as of 2021 May. However, if mechanisms are present that can split comets several tens or even hundreds of astronomical units before perihelion at $\sim 1-10 \mathrm{~m} \mathrm{~s}^{-1}$, it seems plausible that they may, under other circumstances that may or may not apply to $\mathrm{C} / 2021 \mathrm{~A} 1$, propel smaller, unseen fragments or large dust grains from the comet at similar $r$ with the $v_{d} \geqslant v_{0} \sim 0.1-1 \mathrm{~m} \mathrm{~s}^{-1}$ necessary to reach Venus. Without a clear physical explanation for this behavior, however, we cannot meaningfully speculate on the likelihood of such activity contributing substantially to the meteoroid flux at Venus.

\subsection{Meteor Observability}

Venus's MOID point passage near 2021 December 19 21:00 UT, when large, distantly released meteoroids from C/ 2021 A1 are most favored to reach the planet, will occur while Earth is only 0.32 au away. From Earth, Venus will have an apparent diameter of $53^{\prime \prime}$ at $28^{\circ}$ solar elongation with a thin crescent illuminated at a $140^{\circ}$ phase angle. Meteors from the comet will approach the planet at $v_{\infty}=78 \mathrm{~km} \mathrm{~s}^{-1}$ from directions tightly clustered within a few arcminutes of the radiant at R.A. $10^{\mathrm{h}} 52^{\mathrm{m}}$ and decl. $+36^{\circ} 55^{\prime}$ (J2000) prior to gravitational focusing. This radiant is overhead from a subradiant point at longitude $353^{\circ}$ and latitude $+27^{\circ}$, which is on both the Earth-facing and nightside hemispheres near the evening terminator. Figure 7 plots the contours of the radiant zenith angle over the apparent disk and illuminated crescent of Venus viewed from Earth, indicating the portion of the disk where meteors from the comet, if any, are likely to appear.

At a distance of $0.32 \mathrm{au}$, meteors on Venus will be $\sim 28 \mathrm{mag}$ fainter than a terrestrial meteor of the same intrinsic brightness observed from a typical $\sim 100 \mathrm{~km}$ away. A $0.5 \mathrm{~m}$ aperture telescope and detector with a system throughput of $30 \%$ under ideal sky conditions is sensitive to point sources out to $V \sim 16$ 


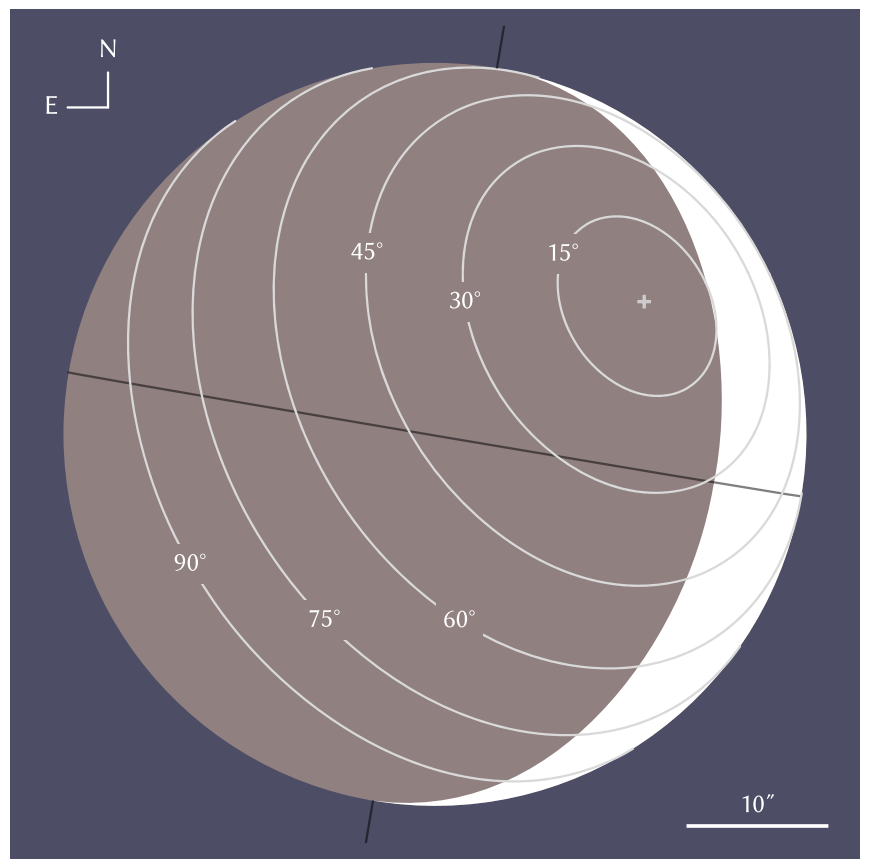

Figure 7. Spatial map for potential meteors on Venus viewed from Earth at 2021 December 19 21:00 UT. Contours indicate curves of constant radiant zenith angle and encircle the subradiant point (marked by a plus sign). Dark lines indicate Venus's equator and axis of rotation, while the white crescent represents the solar-illuminated portion of the planet. Meteors are possible anywhere on the disk that the zenith angle is $<90^{\circ}$ but are most probable near the subradiant point.

in exposures as short as $\sim 0.1 \mathrm{~s}$, corresponding to Venus meteors of $V \sim-12$ from $\sim 100 \mathrm{~km}$ away. While terrestrial meteors from solar system comets are never as fast as the $v_{\infty}=78 \mathrm{~km} \mathrm{~s}^{-1}$ expected for $\mathrm{C} / 2021 \mathrm{~A} 1$ meteors on Venus, Leonid meteors are nearly so, at $v_{\infty}=71 \mathrm{~km} \mathrm{~s}^{-1}$, and so serve as reasonably close analogs, although $\mathrm{C} / 2021 \mathrm{~A} 1$ meteors of the same mass will be $\sim 20 \%$ more energetic and additionally brighter from the shorter scale height in Venus's upper atmosphere (Christou 2004; McAuliffe \& Christou 2006). Leonids of $V \sim-12$ are not uncommon and may be produced by meteoroids of $\sim 0.2 \mathrm{~kg}$ in mass, which have $\beta \sim 10^{-5}$ (Shrbeny` \& Spurny`2009). No dust with $\beta \lesssim 10^{-3.5}$ produced by surface sublimation of $\mathrm{CO}$ can reach Venus, so other mechanisms - such as those responsible for the distant preperihelion splitting of $\mathrm{C} / 1988 \mathrm{~F} 1$ and $\mathrm{C} / 1988 \mathrm{~J} 1$ and the Kreutz sungrazers, as discussed in the previous section-are required to produce any meteors bright enough to be observed on Venus from Earth.

In practice, $V \sim 16$ serves as an optimistic limit, given that any meteors must be observed in close proximity to Venus's brilliantly illuminated dayside, and coronagraphic optics like those developed to search for lightning on the nightside may be required to approach this limit (e.g., Hansell et al. 1995). Bandpass filters encompassing select bright meteor emission features, such as the Na I D lines at $589.0 / 589.6 \mathrm{~nm}$ or the O I line at $777 \mathrm{~nm}$ (Carbary et al. 2004), may further aid in straylight and background rejection. Additionally, the low elongation of Venus means that any observations will be limited to high airmass in twilight, while the short window of no more than $\sim 2 \mathrm{hr}$ for bright meteors associated with distant activity geographically restricts any such observations to a narrow corridor through the Atlantic Ocean and the easternmost portions of Canada and Brazil.
Fluorescence from a meteoritic metallic vapor layer, like that detected by Schneider et al. (2015) on Mars following the C/ 2013 A1 encounter, may also be observable near the terminator - particularly near the northern limb, where the apparent column density is elevated by foreshortening. One appealing candidate is $\mathrm{Na} \mathrm{I}$, whose $\mathrm{D}$ lines are readily observable from the ground and for which a persistent meteoritic layer of $10^{13}-10^{14}$ atoms $\mathrm{m}^{-2}$ is well observed over Earth (e.g., Cabannes et al. 1938; Chapman 1939; Chamberlain 1956). As on Earth, Venus's low heliocentric radial velocity of $-0.2 \mathrm{~km} \mathrm{~s}^{-1}$ during the encounter, combined with the deep $\mathrm{NaI} \mathrm{D}$ Fraunhofer absorption lines in the solar spectrum, strongly suppresses the intensity of the resonance emission, with a fluorescence efficiency of $\sim 1{\text { photon } \mathrm{s}^{-1} \text { atom }}^{-1}$ for the D2 $(589.0 \mathrm{~nm})$ line. Keck HIRES spectroscopy has been demonstrated to be sensitive to emission lines on Venus down to a vertical equivalent of $\sim 20$ rayleighs (Slanger et al. 2001), and other large telescopes equipped with echelle or FabryPérot etalon spectrographs with spectral resolving power $\gtrsim 50,000$ that can cleanly distinguish Venusian emission lines from telluric lines-separated by $8.3 \mathrm{~km} \mathrm{~s}^{-1}$ in radial velocity - may be similarly sensitive. This limit translates to $\sim 2 \times 10^{11}$ atoms $\mathrm{m}^{-2}$ of $\mathrm{NaI}$, or the typical $\mathrm{Na}$ content of $\sim 200 \mathrm{~kg}$ of ablated meteoritic material distributed over one hemisphere of Venus in the absence of ionization or other upper atmospheric chemical reactions that may remove $\mathrm{NaI}$. We note, however, that Venus lacks a persistent Na I layer like Earth's, suggesting that $\mathrm{Na}$ I is indeed rapidly consumed by atmospheric chemistry (Krasnopol'sky 1983). A more careful analysis is required to evaluate if meteoritic $\mathrm{Na}$ I can sufficiently persist for $\gtrsim 1 \mathrm{hr}$ ( i.e., at least through the end of the meteor shower) for any plausible mass of ablated meteoritic material to be detected in this manner.

Additionally, the Akatsuki orbiter remains in operation around Venus (Nakamura et al. 2011) and will likely be the only spacecraft in the immediate vicinity of the planet during the C/2021 A1 encounter; no flybys of Venus are planned for the Parker Solar Probe, ${ }^{6}$ Solar Orbiter, ${ }^{7}$ or BepiColombo ${ }^{8}$ within 1 month of the event. Absent an extreme level of distant activity by unknown mechanisms or severe violations of our defined assumptions, the encounter poses negligible risk to spacecraft health. Even an enormous $\dot{m}_{10} \sim 1000 \mathrm{~kg}$ from CO surface sublimation comparable to that of $\mathrm{C} / 2017 \mathrm{~K} 2$ and $\sim 1000 \times$ larger than inferred for $\mathrm{C} / 2013 \mathrm{~A} 1$ will produce a meteoroid fluence of $\lesssim 10^{-3}$ particles $\mathrm{m}^{-2}$ at Venus for all plausible values of $v_{10}$ and $\sigma^{*}$.

Akatsuki, however, may have the capability to observe any meteors and their subsequent effects on Venus. Of note, Akatsuki carries a lightning and airglow camera (LAC; Takahashi et al. 2018) that monitors for lightning at the $777 \mathrm{~nm}$ O I line that is also a strong meteor emission feature and thus should be sensitive to bright meteors. However, LAC is only operational for 20-30 minutes per 10 days of orbit when the Sun is fully eclipsed by Venus from the spacecraft, so the camera is unlikely to be operable during the brief window of possible meteor activity from C/2021 A1. Akatsuki also carries an ultraviolet imager (UVI; Yamazaki et al. 2018), which includes a $283 \mathrm{~nm}$ channel covering the $\mathrm{Mg}$ II resonance lines at $279.6 / 280.3 \mathrm{~nm}$ that are among the brightest emission

\footnotetext{
6 http://parkersolarprobe.jhuapl.edu/The-Mission/index.php\#Timeline

7 https://sci.esa.int/web/solar-orbiter/-/44181-mission-operations

8 https://sci.esa.int/web/bepicolombo/-/48871-getting-to-mercury
} 
features of terrestrial meteors (Carbary et al. 2004) and were also the brightest lines observed in the C/2013 A1 meteoritic layer on Mars (Schneider et al. 2015). The UVI can integrate for up to $11 \mathrm{~s}$ per exposure and may be useful for directly imaging bright meteors during the encounter, as well as constraining the brightness of any $\mathrm{Mg}$ II layer above the twilit limb afterward, although the wide $14 \mathrm{~nm}$ bandpass means that such observations will likely be strongly impacted by stray light originating from the illuminated dayside if not performed in eclipse. Finally, features in the ionospheric electron density profile have been characterized as meteoritic ion layers (Pätzold et al. 2009; Withers et al. 2013), and Akatsuki's Radio Science (Imamura et al. 2017) radio occultation experiment may be sensitive to large perturbations of these layers comparable to that observed by similar methods during C/2013 A1's encounter with Mars (Restano et al. 2015; Sánchez-Cano et al. 2020).

\section{Conclusions}

The upcoming encounter of $\mathrm{C} / 2021 \mathrm{~A} 1$ with Venus is among the closest known of a long-period comet to any planet and surpassed in recent history only by the 2014 encounter of C/2013 A1 with Mars, which produced a meteor shower on the planet that was indirectly observed by multiple spacecraft. To help ascertain the potential for meteors on Venus, we collected imagery and spectroscopy of C/2021 A1 following its discovery, in advance of the encounter. We found the comet's dust color and size to be typical of other dynamically old longperiod comets at its distance, with the tail optically dominated by $a_{d} \sim 0.1-1 \mathrm{~mm}$ grains produced within the prior year. Our nondetection of $\mathrm{CN}$ sets an upper limit of $\mathrm{CN} / A f \rho<10^{22.5}$ molec s ${ }^{-1} \mathrm{~m}^{-1}$ that is considerably lower than the values typically reported; however, few such measurements have been reported at the $r=4-5$ au of our observations, so the low $\mathrm{CN} /$ Af $\rho$ may not necessarily be abnormal for comets at this distance.

Trajectory analysis shows that-barring an unlikely $v_{d} \gtrsim 0.5$ $\mathrm{km} \mathrm{s}^{-1}$ outburst before the comet reaches $r \approx 2 \mathrm{au}$ in 2021 September-large dust grains of $a_{d} \gtrsim 1 \mathrm{~mm}$ released at $r \gtrsim 30$ au are dynamically most strongly favored to reach Venus, but cometary dust production behavior at such large $r$ is poorly constrained. These large grains inefficiently scatter light, and their presence in the dust trail cannot be usefully constrained telescopically. Quiescent surface sublimation of $\mathrm{CO}$ beginning as far as $r \sim 120$ au can plausibly produce $a_{d} \sim 1 \mathrm{~mm}$ dust grains that become meteors on Venus but likely requires the nucleus to have had a much greater abundance of near-surface $\mathrm{CO}$ than the minute coma we observed suggests is presently accessible. Other distant activity mechanisms, such as those responsible for splitting comet nuclei hundreds of astronomical units away, could potentially produce larger meteors on Venus, the brightest of which may be directly observable from Earth around 2021 December 19 20-22:00 UT. As with C/2013 A1's Mars encounter, the aftermath of a particularly strong meteor shower can also be detected through the formation of a meteoritic metallic vapor layer and perturbations of the ionosphere from Earth and/or by the Akatsuki orbiter around Venus. We encourage observations of this event to take advantage of the rare opportunity to probe an otherwise unseen dust trail and subsequently constrain cometary behavior at distances where no comets have ever been observed.
We thank Michael S. P. Kelley and Lori M. Feaga for help with collecting observations, Ludmilla Kolokolova for discussions on the properties of cometary dust grains, and an anonymous referee for helpful comments and suggestions in a review of this manuscript. We additionally thank Joel Pearman, Kevin Rykoski, and Carolyn Heffner for observing support with the Palomar Hale Telescope, as well as Sydney Perez and Ana Hayslip for their support with the Lowell Discovery Telescope.

This research makes use of observations from the Hale Telescope at Palomar Observatory, which is owned and operated by Caltech and administered by Caltech Optical Observatories.

These results made use of the Lowell Discovery Telescope (LDT) at Lowell Observatory. Lowell is a private, nonprofit institution dedicated to astrophysical research and public appreciation of astronomy and operates the LDT in partnership with Boston University, the University of Maryland, the University of Toledo, Northern Arizona University, and Yale University. The University of Maryland observing team consisted of Quanzhi Ye, James Bauer, Michaela Blain, Adeline Gicquel-Brodtke, Tony Farnham, Lori Feaga, Michael Kelley, and Jessica Sunshine.

This research has made use of data and/or services provided by the International Astronomical Union's Minor Planet Center and by the Jet Propulsion Laboratory's Solar System Dynamics group.

This work was supported by NSF award AST1852589. S.V. is supported by an NSF Graduate Research Fellowship and the Paul \& Daisy Soros Fellowship for New Americans.

Facilities: DCT (LMI), Hale (DBSP, WIRC).

Software: Astropy (Astropy Collaboration et al. 2013), emcee (Foreman-Mackey et al. 2013), Matplotlib (Hunter 2007), NumPy (Van Der Walt et al. 2011), PypeIt (Prochaska et al. 2020), sbpy (Mommert et al. 2019).

\section{ORCID iDs}

Qicheng Zhang (1D https://orcid.org/0000-0002-6702-191X Quanzhi Ye

(叶泉 志) (DiD https://orcid.org/0000-0002-4838-7676

Shreyas Vissapragada (1) https://orcid.org/0000-00032527-1475

Matthew M. Knight (iD https://orcid.org/0000-0003-2781-6897 Tony L. Farnham (iD https://orcid.org/0000-0002-4767-9861

\section{References}

A'Hearn, M. F., Millis, R. C., Schleicher, D. G., Osip, D. J., \& Birch, P. V. 1995, Icar, 118, 223

A’Hearn, M. F., Schleicher, D., Millis, R., Feldman, P., \& Thompson, D. 1984, AJ, 89, 579

Astropy Collaboration, Robitaille, T. P., Tollerud, E. J., et al. 2013, A\&A, 558, A33

Beech, M. 1998, MNRAS, 294, 259

Benna, M., Mahaffy, P., Grebowsky, J., et al. 2015, GeoRL, 42, 4670

Boe, B., Jedicke, R., Meech, K. J., et al. 2019, Icar, 333, 252

Cabannes, J., Dufay, J., \& Gauzit, J. 1938, ApJ, 88, 164

Carbary, J., Morrison, D., Romick, G., \& Yee, J. 2004, AdSpR, 33, 1455

Chamberlain, J. W. 1956, JATP, 9, 73

Chambers, K., Magnier, E., Metcalfe, N., et al. 2016, arXiv:1612.05560

Chapman, S. 1939, ApJ, 90, 309

Christou, A. 2010, MNRAS, 402, 2759

Christou, A. A. 2004, Icar, 168, 23

Combi, M. R., Mäkinen, T., Bertaux, J.-L., et al. 2019, ApJL, 884, L39

Crismani, M. M., Schneider, N. M., Plane, J. M., et al. 2017, NatGe, 10, 401

Cui, X.-Q., Zhao, Y.-H., Chu, Y.-Q., et al. 2012, RAA, 12, 1197 
DiSanti, M., Bonev, B., Gibb, E., et al. 2016, ApJ, 820, 34

Farnham, T. L., Schleicher, D. G., \& A'Hearn, M. F. 2000, Icar, 147, 180

Farnham, T. L., Schleicher, D. G., Woodney, L. M., et al. 2001, Sci, 292, 1348

Farnocchia, D., Chesley, S. R., Chodas, P. W., et al. 2014, ApJ, 790, 114

Farnocchia, D., Chesley, S. R., Micheli, M., et al. 2016, Icar, 266, 279

Feldman, P. D., Weaver, H. A., A'Hearn, M. F., Combi, M. R., \& Russo, N. D. 2018, AJ, 155, 193

Finson, M., \& Probstein, R. 1968, ApJ, 154, 327

Foreman-Mackey, D., Hogg, D. W., Lang, D., \& Goodman, J. 2013, PASP, 125,306

Fray, N., Bénilan, Y., Cottin, H., Gazeau, M.-C., \& Crovisier, J. 2005, P\&SS, 53, 1243

Fulle, M. 2004, in Comets II, ed. M. C. Festou, H. U. Keller, \& H. A. Weaver (Tucson, AZ: Univ. Arizona Press), 565

Gaia Collaboration, Brown, A. G., Vallenari, A., et al. 2021, A\&A, 649, A1

Hänni, N., Altwegg, K., Pestoni, B., et al. 2020, MNRAS, 498, 2239

Hansell, S., Wells, W., \& Hunten, D. 1995, Icar, 117, 345

Haser, L. 1957, BSRSL, 43, 740

Hui, M.-T., Jewitt, D., \& Clark, D. 2017, AJ, 155, 25

Hunter, J. D. 2007, CSE, 9, 90

Imamura, T., Ando, H., Tellmann, S., et al. 2017, EP\&S, 69, 137

Jenniskens, P., Lauretta, D. S., Towner, M. C., et al. 2021, Icar, 365, 114469

Jewitt, D. 2015, AJ, 150, 201

Jewitt, D., Agarwal, J., Hui, M.-T., et al. 2019, AJ, 157, 65

Jewitt, D., Kim, Y., Mutchler, M., et al. 2021, AJ, 161, 188

Jewitt, D., Luu, J., \& Chen, J. 1996, AJ, 112, 1225

Jewitt, D., \& Matthews, H. 1999, AJ, 117, 1056

Jones, G. H., Knight, M. M., Battams, K., et al. 2018, SSRv, 214, 20

Kelley, M. S., Farnham, T. L., Bodewits, D., Tricarico, P., \& Farnocchia, D. 2014, ApJL, 792, L16

Kizner, W. 1961, P\&SS, 7, 125

Knight, M. M., \& Schleicher, D. G. 2014, AJ, 149, 19

Kolokolova, L., Nagdimunov, L., \& Mackowski, D. 2018, JQSRT, 204, 138

Krasnopol'sky, V. 1983, in Venus, ed. D. M. Hunten, L. Colin, \& T. M. Donahue (Tucson, AZ: Univ. Arizona Press), 459

Kresak, L. 1993, A\&A, 279, 646

Królikowska, M., \& Dybczyński, P. A. 2017, MNRAS, 472, 4634

Lasue, J., Levasseur-Regourd, A. C., Hadamcik, E., \& Alcouffe, G. 2009, Icar, 199,129

Leonard, G., Aschi, S., Pettarin, E., et al. 2021a, MPEC, 2021, A99

Leonard, G., Aschi, S., Pettarin, E., et al. 2021b, CBET, 2021, 4907

Li, J., \& Jewitt, D. 2015, AJ, 149, 133

Li, J.-Y., Samarasinha, N. H., Kelley, M. S., et al. 2014, ApJL, 797, L8

Li, J.-Y., Samarasinha, N. H., Kelley, M. S., et al. 2016, ApJL, 817, L23

Lyytinen, E., \& Jenniskens, P. 2003, Icar, 162, 443

Marsden, B. 1988, BAAS, 20, 898

Massey, P., Dunham, E., Bida, T., et al. 2013, AAS Meeting, 221, 345
McAuliffe, J. P., \& Christou, A. A. 2006, Icar, 180, 8

Meech, K., \& Svoren, J. 2004, in Comets II, ed. M. C. Festou, H. U. Keller, \& H. A. Weaver (Tucson, AZ: Univ. Arizona Press), 317

Meech, K. J., Kleyna, J. T., Hainaut, O., et al. 2017, ApJL, 849, L8

Meftah, M., Damé, L., Bolsée, D., et al. 2018, A\&A, 611, A1

Miles, R. 2013, JBAA, 123, 363

Mommert, M., Kelley, M., de Val-Borro, M., et al. 2019, JOSS, 4, 1426

Moorhead, A. V., Wiegert, P. A., \& Cooke, W. J. 2014, Icar, 231, 13

Nakamura, M., Imamura, T., Ishii, N., et al. 2011, EP\&S, 63, 443

Ohtsuka, K. 1991, in Origin and Evolution of Interplanetary Dust, ed.

A. C. Levasseur-Regourd \& H. Hasegawa (Berlin: Springer), 315

Oke, J., \& Gunn, J. 1982, PASP, 94, 586

Opitom, C., Guilbert-Lepoutre, A., Jehin, E., et al. 2016, A\&A, 589, A8

Pätzold, M., Tellmann, S., Häusler, B., et al. 2009, GeoRL, 36, 5203

Prochaska, J. X., Hennawi, J. F., Westfall, K. B., et al. 2020, JOSS, 5, 2308

Rauer, H., Arpigny, C., Boehnhardt, H., et al. 1997, Sci, 275, 1909

Restano, M., Plaut, J. J., Campbell, B. A., et al. 2015, GeoRL, 42, 4663

Rousselot, P. 2008, A\&A, 480, 543

Russo, N. D., Vervack, R., Jr., Weaver, H., et al. 2008, ApJ, 680, 793

Sánchez-Cano, B., Lester, M., Witasse, O., et al. 2020, JGRA, 125, e27344

Schleicher, D. G., \& Bair, A. N. 2011, AJ, 141, 177

Schleicher, D. G., Woodney, L. M., \& Birch, P. V. 2002, Cometary Science after Hale-Bopp (Berlin: Springer), 401

Schneider, N. M., Deighan, J., Stewart, A., et al. 2015, GeoRL, 42, 4755

Sekanina, Z., \& Chodas, P. W. 2004, ApJ, 607, 620

Sekanina, Z., \& Kracht, R. 2014, arXiv:1404.5968

Sekanina, Z., \& Kracht, R. 2016, ApJ, 823, 2

Shrbeny’, L., \& Spurny`, P. 2009, A\&A, 506, 1445

Skrutskie, M., Cutri, R., Stiening, R., et al. 2006, AJ, 131, 1163

Slanger, T., Cosby, P., Huestis, D., \& Bida, T. 2001, Sci, 291, 463

Sykes, M. V., \& Walker, R. G. 1992, Icar, 95, 180

Takahashi, Y., Sato, M., Imai, M., et al. 2018, EP\&S, 70, 88

Tricarico, P., Samarasinha, N. H., Sykes, M. V., et al. 2014, ApJL, 787, L35

Trigo-Rodríguez, J., García-Melendo, E., Davidsson, B., et al. 2008, A\&A, 485,599

Valsecchi, G. B., Milani, A., Gronchi, G. F., \& Chesley, S. R. 2003, A\&A, 408, 1179

Van Der Walt, S., Colbert, S. C., \& Varoquaux, G. 2011, CSE, 13, 22

Vaubaillon, J., Maquet, L., \& Soja, R. 2014, MNRAS, 439, 3294

Wilson, J. C., Eikenberry, S. S., Henderson, C. P., et al. 2003, Proc. SPIE, 4841, 451

Withers, P., Christou, A., \& Vaubaillon, J. 2013, AdSpR, 52, 1207

Yamazaki, A., Yamada, M., Lee, Y. J., et al. 2018, EP\&S, 70, 23

Yang, B., Jewitt, D., Zhao, Y., et al. 2021, ApJL, 914, L17

Ye, Q., Knight, M. M., Kelley, M. S., et al. 2021, PSJ, 2, 23

Ye, Q.-Z., Brown, P. G., Bell, C., et al. 2015, ApJ, 814, 79

Ye, Q.-Z., \& Hui, M.-T. 2014, ApJ, 787, 115 